\title{
Insight into the Origin of Chiral-Induced Spin Selectivity from a Symmetry Analysis of Electronic Transmission
}

\author{
Martin Sebastian Zöllner, Solmar Varela, Ernesto Medina, Vladimiro Mujica, \\ and Carmen Herrmann* \\ Department of Chemistry, University of Hamburg, Germany \\ E-mail: carmen.herrmann@chemie.uni-hamburg.de
}

\begin{abstract}
The chiral-induced spin selectivity (CISS) effect, which describes the spin-filtering ability of diamagnetic structures like DNA or peptides having chiral symmetry, has emerged in the past years as the central mechanism behind a number of important phenomena, like long-range biological electron transfer, enantiospecific electrocatalysis, and molecular recognition. Also, CISS-induced spin polarization has a considerable promise for new spintronic devices and the design of quantum materials. The CISS effect is attributed to spin-orbit coupling, but a sound theoretical understanding of the surprising magnitude of this effect in molecules without heavy atoms is currently lacking. We are taking an essential step into this direction by analyzing the importance of imaginary terms in the Hamiltonian as a necessary condition for non-vanishing spin polarization in helical structures. Based on first-principles calculations and analytical considerations, we perform a symmetry analysis of the key quantities determining transport probabilities of electrons of different spin orientations. These imaginary terms originate from the spin-orbit coupling, and they preserve the Hermitian nature of
\end{abstract}


the Hamiltonian. Hence, they are not related to the breaking of time-reversal symmetry resulting from the fact that molecules are open systems in a junction. Our symmetry analysis helps to identify essential constraints in the theoretical description of the CISS effect. We further draw an analogy with the appearance of imaginary terms in simple models of barrier scattering, which may help understanding the unusually effective long-range electron transfer in biological systems. 


\section{Introduction}

A substantial body of experimental results and theoretical work has established on firm ground that electron transport, electron transfer or bond polarization in extended chiral systems translates into electron spin polarization. ${ }^{1}$ The phenomenon, know as Chiral-Induced Spin Selectivity (CISS), is so pervasive that its effects have been established in local probe measurements, e.g. scanning tunneling and atomic force microscopy (STM and AFM), in molecular junctions; in electron photoemission through chiral monolayers; ${ }^{2}$ in electrode chemical reactions ${ }^{3}{ }^{[-6}$ and in enantio-selective response to magnetic polarization. ${ }^{7}$ It was observed in DNA,,$\frac{810}{10}$ proteins, $\frac{11}{2}$ oligopeptides,,$\frac{12113}{}$ and helicenes. $\frac{14115}{}$ None of these structures feature any intrinsic magnetization, so the spin filtering must result from symmetry breaking associated with the electronic structure of chiral molecules ${ }^{1}$ or external probes in the transport configuration ${ }^{16}$. The effect is important not only from a fundamental science per-

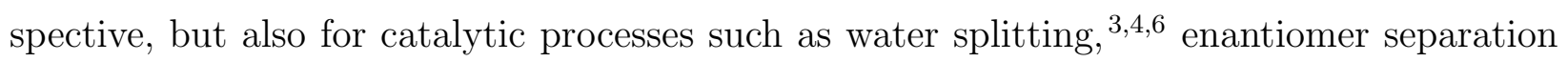
and spintronic devices. 17

Based on a wide range of analytical and tight-binding theoretical analyses, $\frac{18[26}{26}$ is now broadly accepted that CISS is related to a combination of enhanced spin-orbit coupling (SOC) connected to breaking of spatial inversion symmetry, similar to that present in Rashba spin-orbit coupling ${ }^{27}$ observed, e.g., in metal surfaces ${ }^{28 \mid 29}$ and semiconductor nanosheets. 30 Given that it was observed in diamagnetic molecules consisting uniquely of light atoms, e.g. carbon, hydrogen, oxygen and nitrogen, where spin-orbit coupling is weak, the magnitude of this CISS effect was (and still is) puzzling. To address this question from an ab initio perspective, and for establishing structure-property relationships later on, a first-principles approach is highly needed.

The CISS effect was the focus of several previous theoretical investigations. The first atomistic approaches to describe CISS theoretically used tight-binding models with ad-hoc effective parameters defining the Hamiltonian. Regarding the CISS effect, the first estimate for a tight-binding Hamiltonian and its parameters based on a semi-empirical methodology 
was done by Medina et al., 19131 showing an enhancement of the spin-orbit coupling for helical systems in analogy to the increased spin-orbit coupling in carbon nanotubes compared to graphene. Later, Maslyuk et al. demonstrated that a helical structure leads to an enhancement factor in spin polarization of $10^{2}-10^{3}$ compared to a planar structure, $\stackrel{23}{\text { using a }}$ first-principles approach for the first time. However, none of the theoretical works published so far have been able to explain the astonishing magnitude of the CISS effect, but rather underestimate it by several orders of magnitude. It is therefore crucial to look deeper into the relation between spin polarization and the underlying electronic structure to identify what is missing in current theoretical approaches.

To this end, we start by approximating the full Hermitian Hamiltonian matrix as a singleparticle effective matrix built with real orbitals as in conventional density functional theory. In the absence of spin-orbit interaction, this matrix (the Fock matrix) is real symmetric. Adding the spin-orbit interactions results in the appearance of an imaginary term, while preserving the hermiticity of the Fock matrix. We discuss the effect of the spin-orbit term on the symmetry of the Green's function matrices, which in turn determines the electron propagation in the Landauer-Buttiker formulation. In our work, the influence of matrix symmetries on spin polarization will be shown analytically, and the effect of the molecular structure of the described system will be studied numerically for (1) a linear and (2) a helical carbon chain.

We furthermore show an analogy between spin polarization in a Landauer transmission formalism and in barrier scattering, where spin-orbit coupling strongly modifies the length dependence of electron transfer. Through this combined approach, we hope to provide a mechanism for polarization enhancement in electron transport through helical structures, but also into the remarkable efficiency of electron transport in biological structures over long distances. 


\section{The link between chirality-dependent spin polariza- tion and the imaginary part of the effective one- electron Hamiltonian matrix}

To investigate the spin polarization of a current within a molecular junction, we take a closer look at the mathematical formulation of electron transport and the consequences of spinorbit coupling on it. A widely used and well-established approach to describe such electron transport in the coherent tunneling regime is the Landauer-Imry-Büttiker approach. Tunneling is the dominant transport mechanism for the short oligopeptides for which CISS has been studied experimentally, $\underline{3233}$ and for helicene (the description of transport for longer molecules, e.g segments of DNA, $\frac{34}{2}$ requires the inclusion of incoherent transport). This approach has been combined with theories which describe electronic structures in an effective single-particle picture, such as Kohn-Sham density functional theory (KS-DFT) and semi-empirical and tight-binding models. Despite the shortcomings of such single-particle approaches, they have been very successful in understanding single-molecule conductance. 35 Also, DFT was found to quantitatively describe Rashba-type splitting in, e.g., gold surface states $^{28}$ (for tight-binding approaches, compare Ref., ${ }^{36}$ which suggests DFT to be in principle able to describe CISS. Combinations of the Landauer approach and effective single-particle theories have indeed been used to investigate the CISS effect, both within tight-binding

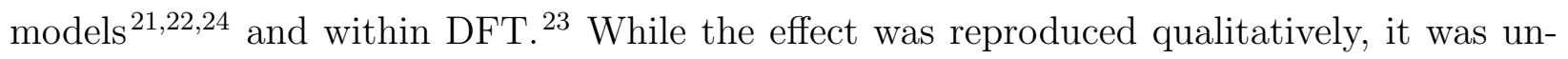
derestimated by several orders of magnitude. To understand what is lacking in these state-ofthe-art theoretical approaches, it is essential to understand how spin polarization originates and, consequently, how it might be enhanced.

In the following, we will show analytically how direction-dependent spin polarization enters the Landauer approach due to imaginary terms induced by spin-orbit coupling. Subsequently, an analysis of effective single-particle Hamiltonian matrices calculated with DFT will confirm the importance of imaginary terms for the description of CISS using first-principles 
methods.

\subsection{Electron transport considering spin-orbit coupling}

In the Landauer-Imry-Büttiker approach,$\frac{37}{37}$ the current $I_{R L}$ through an electrode--moleculeelectrode junction from right to left is evaluated by integrating over a transmission function ${ }^{1}$ $T_{R L}$,

$$
I_{R L}=\int_{E-\frac{e V}{2}}^{E+\frac{e V}{2}} T_{R L}(E) d E
$$

where the transmission function is obtained as

$$
T_{R L}=\operatorname{Tr}\left[\boldsymbol{\Gamma}_{L} \mathbf{G} \boldsymbol{\Gamma}_{R} \mathbf{G}^{\dagger}\right]
$$

The central (scattering) region's Green's function $\mathbf{G}$ at any energy $E$ can be calculated by partitioning the full effective single-particle Hamiltonian matrix $\mathbf{H}$ and the full overlap matrix $\mathbf{S}$ into a central, left- and right-electrode region, as

$$
\mathbf{G}=\frac{1}{E \mathbf{S}_{C}-\mathbf{H}_{C}-\mathbf{\Sigma}_{L}-\mathbf{\Sigma}_{R}}
$$

with $\mathbf{H}_{C}$ as the effective single-particle Hamiltonian matrix of the central region, $\mathbf{S}_{C}$ as the overlap matrix of the central region, and $\boldsymbol{\Sigma}_{L}$ and $\boldsymbol{\Sigma}_{R}$ as the self-energies of the left and right electrodes. The central region can comprise the molecule only or an "extended molecule" which also includes parts of the electrodes. The effective single-particle Hamiltonian (in the following simply referred to as "Hamiltonian") can result from electronic structure theories such as Kohn-Sham density functional theory, Hartree-Fock theory, sempiempirical methods or Hückel / tight binding model: ${ }^{2}$.

\footnotetext{
${ }^{1}$ This equation is strictly valid at a temperature of $0 \mathrm{~K}$, but it is also a good approximation at higher temperatures.

${ }^{2}$ Kohn-Sham density functional theory is a many-body description where actual electrons are replaced by fermionic quasi-particles. While this leaves open fundamental questions on the relation between these
} 


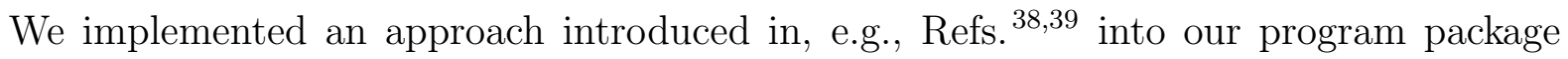
ARTAIOS $^{40 \mid 41}$ to investigate the effect of spin-orbit coupling on the transmission function and to take into account the possibility of spin flips during electron transport. This ansatz is based on a two-component Kohn-Sham DFT description of the electronic structure, where all orbitals have a spin-up $(\alpha, \uparrow)$ and a spin-down $(\beta, \downarrow)$ component. Since spin polarization in the experiment occurs with respect to electron spins in or against transport direction, we choose the $z$ axis, corresponding to the transport direction, for spin quantization. When representing effective single-particle operators $\hat{o}$ in a set of atom-centered basis functions $\left\{\chi_{\mu}\right\}$, all matrices $\mathbf{M}$ assume a $2 \times 2$ structure of the type

$$
\mathbf{M}=\left(\begin{array}{cc}
\mathbf{M}^{\uparrow \uparrow} & \mathbf{M}^{\uparrow \downarrow} \\
\mathbf{M}^{\downarrow \uparrow} & \mathbf{M}^{\downarrow \downarrow}
\end{array}\right)
$$

where $\mathbf{M}^{\sigma \sigma^{\prime}}$ denotes a block of matrix elements $\mathbf{M}_{\mu \nu}^{\sigma \sigma^{\prime}}=\left\langle\chi_{\mu}^{\sigma}|\hat{o}| \chi_{\nu}^{\sigma \prime}\right\rangle$ with the two basis functions referring to electrons of spin orientation $\sigma$ and $\sigma^{\prime}$, respectively $\left(\sigma, \sigma^{\prime} \in\{\uparrow, \downarrow\}\right)$. The transmission function in this two-component approach is a sum of four different contributions (two spin-flip and two spin-conserving ones, for details see Section C),

$$
T=T_{R L}^{\uparrow \uparrow}+T_{R L}^{\downarrow \uparrow}+T_{R L}^{\uparrow \downarrow}+T_{R L}^{\downarrow \downarrow}
$$

From this, the spin polarization $P$ (i.e., the normalized difference in transmission between spin-up and spin-down electrons) can be evaluated as

$$
P=\frac{T_{R L}^{\uparrow \uparrow}+T_{R L}^{\downarrow \uparrow}-T_{R L}^{\uparrow \downarrow}-T_{R L}^{\downarrow \downarrow}}{T}
$$

This is an energy-dependent quantity, with the value at the Fermi energy giving an estimate of measured spin polarization at small bias voltages.

fermions and measurable properties, in practice transport calculations from KS-DFT have proven very successful for understanding molecular conductance. 
To learn about the origin of the chirality-dependent spin filtering, we take a closer look at the symmetries of the matrices used to calculate the transmission function. We show that these symmetries can be broken as spin-orbit coupling leads to imaginary parts in the Hamiltonian matrix, and evaluate the resulting consequences for spin polarization.

\subsection{Symmetry breaking by imaginary terms introduced by spin- orbit coupling}

To analyze under which circumstances the spin polarization of the transmitted electrons can be different from zero, we focus on the symmetry properties of the Green's function $\mathbf{G}$ and the resulting symmetries of the transmission function. Time-reversal and spatial-inversion symmetry translate into the following properties of the transmission function,

$$
\begin{array}{lll}
T_{R L}^{\uparrow \uparrow}=T_{L R}^{\uparrow \uparrow} \text { and } & T_{R L}^{\uparrow \downarrow}=T_{L R}^{\downarrow \uparrow} & \text { (spatial-inversion symmetry) } \\
T_{R L}^{\uparrow \uparrow}=T_{L R}^{\downarrow \downarrow} \text { and } & T_{R L}^{\uparrow \downarrow}=T_{L R}^{\uparrow \downarrow} & \text { (time-reversal symmetry). }
\end{array}
$$

If both apply, we can formulate the relation

$$
T_{R L}^{\uparrow \uparrow}=T_{R L}^{\downarrow \downarrow}=T_{L R}^{\downarrow \downarrow}=T_{L R}^{\uparrow \uparrow},
$$

and no spin polarization can occur.

Time-reversal symmetry as defined in Eq. (8) (also compare Ref. ${ }^{[36}$ ) will hold as long as there is no external magnetic field (as is the case in all CISS experiments so far) ${ }^{3}$. In the Landauer transport formulation, a different type of time-reversal symmetry breaking arises

\footnotetext{
${ }^{3}$ It can be shown that when time-reversal symmetry is maintained, Kramer's degeneracy applies. ${ }^{42}$ This leads to zero spin polarization in thermodynamic equilibrium. $\stackrel{43}{ }$ When a bias voltage is applied, this selects one of the Kramers pairs, opening up the possibility of spin polarization. Also note that previous work showing that spin polarization is not possible when considering leads with one transport mode in twoterminal setups ${ }^{44}$ are not directly transferable to our work, since the realistic modeling of molecular junctions as provided by first-principles descriptions allows for multiple transport channels. Similar conclusions can result from considering that spin polarization arises from spin accumulation in the electrodes once a transport direction has been selected by the experimental bias.
} 
from the fact that the molecule in a molecular junction is an open system connected to electrodes acting as reservoirs. A formulation using a circuit-model analysis ${ }^{45}$ has recently appeared in the literature, where the claim is made that spin polarization cannot be detected in two-terminal devices. This result is valid only if the self-energy term in the LandauerButtiker formulation is neglected, which is not the case here.

We find the result that time reversal symmetry (in the sense of Eq. (8D) and spatial inversion symmetry for the transmission functions for a closed-shell system is maintained if $\mathbf{G}$ is either symmetric,

$$
\mathbf{G}=\mathbf{G}^{T}
$$

or Hermitian,

$$
\mathbf{G}=\mathbf{G}^{\dagger}
$$

(for details, see Section $\mathrm{D}$ and $\mathrm{E}$ in the Appendix). For a DFT calculation without spin-orbit coupling (and employing real orbitals), both the effective single-particle Hamiltonian matrix of the scattering region $\mathbf{H}_{C}$ and $\mathbf{G}$ are symmetric, and thus no spin polarization is possible (see Section D).

Spin-orbit coupling adds real (spin-flip) and imaginary parts (spin-flip and spin-conserving) to $\mathbf{H}_{C} \underline{46147}$ (see Section $\mathrm{B}$ in the Appendix). The resulting $\mathbf{H}_{C}$ remains Hermitian but is not symmetric anymore. $\mathbf{S}_{C}$ remains purely real, and the symmetry properties of the two matrices can be written as

$$
\begin{gathered}
\mathbf{H}_{C}=\mathbf{H}_{C}^{\dagger} \neq \mathbf{H}_{C}^{T} \\
\mathbf{S}_{C}=\mathbf{S}_{C}^{\dagger}=\mathbf{S}_{C}^{T} .
\end{gathered}
$$


In contrast, $\boldsymbol{\Sigma}_{\boldsymbol{L}}$ and $\boldsymbol{\Sigma}_{\boldsymbol{R}}$ are not Hermitian $\underline{48}$ — in our approach, they are purely imaginary and symmetric (where we enforce them to have collinear spin structures such that the spin orientations of incoming and transmitted electrons are well defined, see Section A). Therefore, we can write

$$
\boldsymbol{\Sigma}_{L}=\mathrm{i} \operatorname{Im}\left(\boldsymbol{\Sigma}_{L}\right)=\mathrm{i}\left[\operatorname{Im}\left(\boldsymbol{\Sigma}_{L}\right)\right]^{\mathrm{T}}
$$

(and analogously for the right electrode). The presence of imaginary components in $\mathbf{H}_{C}$, combined with its Hermicity and with $\Sigma_{L}$ and $\Sigma_{R}$ being symmetric, can be identified as responsible for spin polarization of transmitted electrons in closed-shell systems: If spinorbit coupling is considered in the calculation of $\mathbf{H}_{C}$, the resulting $\mathbf{G}$ is in general neither Hermitian,

$$
\mathbf{G}=\frac{1}{E \mathbf{S}-\mathbf{H}_{C}-\mathrm{i} \operatorname{Im}\left[\boldsymbol{\Sigma}_{L}\right]-\mathrm{i} \operatorname{Im}\left[\boldsymbol{\Sigma}_{R}\right]} \neq \mathbf{G}^{\dagger}=\frac{1}{E \mathbf{S}-\mathbf{H}_{C}+\mathrm{i} \operatorname{Im}\left[\boldsymbol{\Sigma}_{L}\right]+\mathrm{i} \operatorname{Im}\left[\boldsymbol{\Sigma}_{R}\right]}
$$

nor symmetric,

$$
\mathbf{G}=\frac{1}{E \mathbf{S}-\mathbf{H}_{C}-\mathrm{i} \operatorname{Im}\left[\boldsymbol{\Sigma}_{L}\right]-\mathrm{i} \operatorname{Im}\left[\boldsymbol{\Sigma}_{R}\right]} \neq \mathbf{G}^{T}=\frac{1}{E \mathbf{S}-\mathbf{H}_{C}^{*}-\mathrm{i} \operatorname{Im}\left[\boldsymbol{\Sigma}_{L}\right]-\mathrm{i} \operatorname{Im}\left[\boldsymbol{\Sigma}_{R}\right]}
$$

leading to a loss of the spatial-inversion symmetry in the transmission functions, and to the relation $T_{R L}^{\uparrow \uparrow}=T_{R L}^{\downarrow \downarrow}$ no longer being enforced. This opens the door for spin polarization (for details, see Section $\mathrm{E}$ in the appendix). In other words, by having an imaginary part in the Hamiltonian matrix, the sign of spin polarization can depend on the direction of the transport or, equivalently, on the helicity for a given transport direction (since helicity leads to sign changes of imaginary parts of $\mathbf{H}_{C}$, see Section 3.3.1 below). 


\section{Insights from first-principles electron transport for ideal carbon helices}

In the previous section, we have shown the possibility of helicity- or direction-dependent spin polarization by including spin-orbit coupling in the Landauer formalism. It remains open how the imaginary parts of the Hamiltonian matrix can help us understand which parts of the structure are responsible for spin polarization. To gain insight into this question, we calculated the transmission and the polarization of the transmitted electrons for ideal carbonbased helical and linear chains using DFT. For the helical structures, we would expect the polarization to change its sign upon changing the handedness of the helix, while for the linear chains, there should be no polarization at all. We discuss the resulting polarization in the light of the imaginary part of the Hamiltonian matrix, since this is the source of nonzero polarization. For simplicity, we focus on the spin-conserving parts (note that it is also the spin-conserving transmission that dominates polarization). The transmission functions will be shown in the Supporting Information for completeness.

\subsection{Molecular structures}

The helical molecular structures were created by placing 40 carbon atoms, evenly spaced $(1.3 \AA$ ), along a perfect helical path with a pitch of $3 \AA$ and a radius of $2 \AA$ (see Fig. 1 ). The

linear structures were built by placing carbon atoms evenly spaced (1.3 $\AA$ ) along a perfect linear path. Two linear molecules were investigated (20 and 40 carbon atoms) to make sure the calculated polarization increases with length just as in the experiment. Two hydrogen atoms were added at each of the ends, resulting in an overall cumulene-like structure. The hydrogen atoms used for the saturation were structurally relaxed, while the helical carbon backbone was kept fixed. The relaxed structures were placed between two gold clusters of 10 atoms each, mimicking the electrodes, so that the terminal carbon atoms were in fcc adsorption positions with gold-carbon distances of $2.48 \AA$. The central (scattering) region 
was defined to be the helical/linear molecule only.

It is clear that with a gold-molecule-gold junction in the absence of external magnetic fields, one would not detect any spin polarization experimentally. For this, at least one magnetic electrode would be required (e.g., a nickel tip in the scanning tunneling microscope, 12 for which conductance would be probed with two different orientations of its magnetization, in transport direction and opposite to transport direction). However, we can mimick the effect of this magnetic electrode, i.e., letting through preferentially electrons of a given spin orientation, by considering transmission of spin-up and spin-down electrons separately and evaluating their (normalized) difference according to Eq. (6). The advantage of this approach is that we do not introduce spin polarization on the molecule itself, so that all polarization in the resulting transmission can be attributed uniquely to spin-orbit coupling ${ }^{4}$.
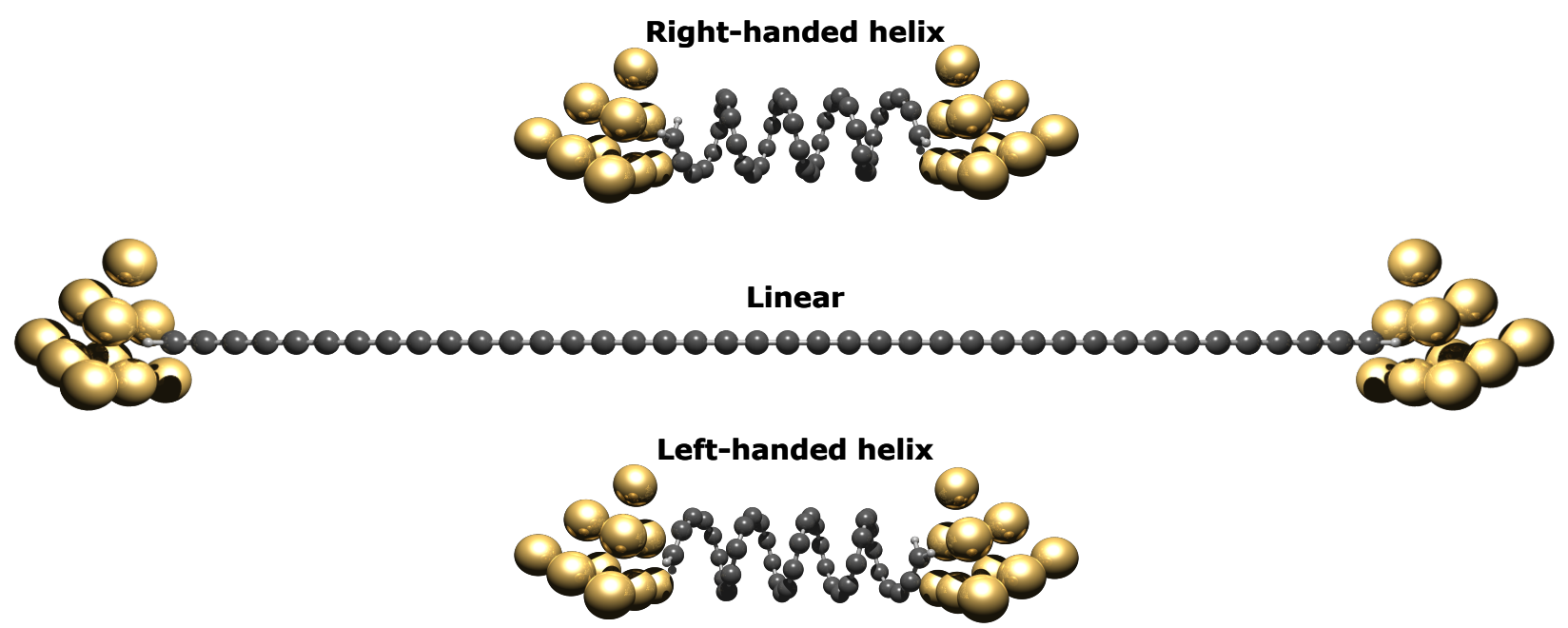

Figure 1: Gold-molecule-gold junctions as used in the DFT calculations consisting of a molecule placed between two 10-atomic gold clusters. Left-handed helix (lower system), right-handed helix (upper system), and the 40-atomic chain (middle system) are depicted.

\footnotetext{
${ }^{4}$ Similar systems were already investigated by Cuniberti et al., ${ }^{23}$ using a different methodology and without analyzing the origin of the spin polarization within the Hamiltonian. A short discussion concerning the deviations of our data from these results is given in the Supporting Information.
} 


\subsection{Polarization of transmitted electrons}

To discuss the energy-dependent polarization of the transmitted electrons, we will focus on the most significant extremum of the polarization, $P_{\text {extr }}$. For the left-handed helix, $P_{\text {extr }}$ has a positive sign $(1.06 \%)$, while for the right-handed helix, the sign is negative $(-1.05 \%)$. As with $P_{\text {extr }}$, the polarization changes its sign for any energy upon changing the handedness of the helix (see Figure 2b)). Reducing the length of the helix leads to a decrease of the spin polarization, as to be expected for the CISS effect (see Supporting Information). For both linear molecules, the polarization is close to zero for any energy value (see Figure 2a)). This is consistent with the expectations for the CISS effect.

The underlying transmission functions are shown in the Supporting Information. Importantly, we find both overall transmission and polarization to be dominated by the two spin-conserving contributions to the transmission, while the spin-flip components play a negligible role. This is in line with previous theoretical work based on DFT ${ }^{23}$ and on macroscopic rate equations. $\underline{49}$
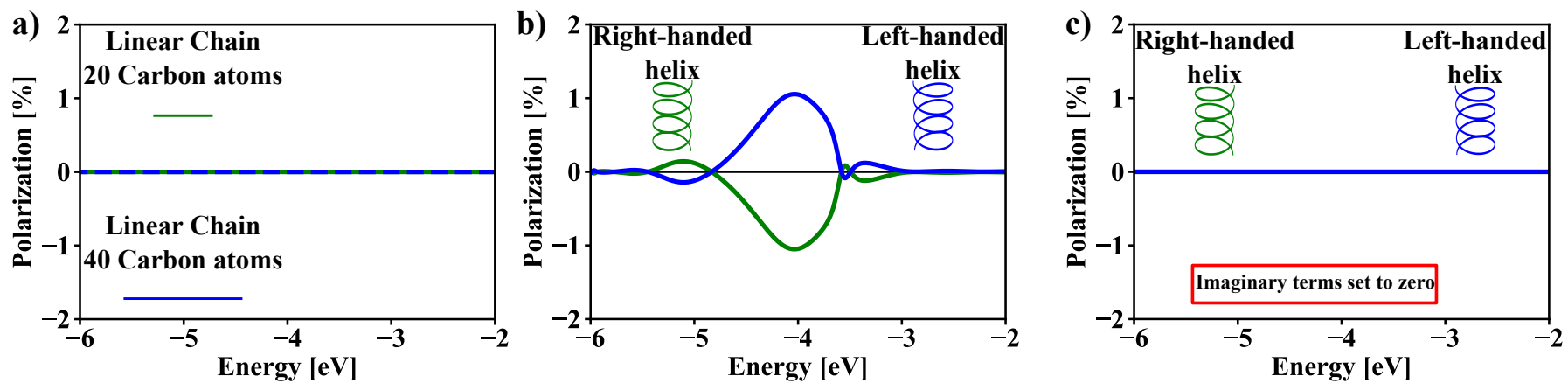

Figure 2: Calculated polarization of the transmitted electrons in the range of $-6.00 \mathrm{eV}$ to $-2.00 \mathrm{eV}$, using the B3LYP exchange-correlation functional. The linear molecules do not show significant polarization, while for the helical ones the polarization can reach absolute values of about $1 \%$. The sign of the polarization changes upon changing the handedness of the helix. If the calculations are done without the imaginary part of the Hamiltonian matrix, the polarization becomes nearly zero for the whole calculated energy range.

Since we suggested that polarization originates from the imaginary parts of the Hamiltonian matrix $\operatorname{Im}\left(\mathbf{H}_{C}\right)$, we additionally recalculated the polarization for the helical molecules 
setting $\operatorname{Im}\left(\mathbf{H}_{C}\right)$ to zero. The results confirm the importance of $\operatorname{Im}\left(\mathbf{H}_{C}\right)$, since the polarization completely vanishes (see Figure 22c). Nevertheless, the amount of spin polarization is much smaller compared to the experiments, suggesting that some contributions to CISS for helical structures are missing in the used approach. We therefore focus on understanding the mechanism leading to the absence of spin polarization for the linear molecules (the linear molecules also have a non-zero $\left.\operatorname{Im}\left(\mathbf{H}_{C}\right)\right)$ and the changing sign of spin polarization comparing the left- and right-handed helices. Both will be investigated next.

\subsection{Origin of polarization}

The presence of CISS for cumulene-like structures within DFT in combination with the Landauer approach was shown by Cuniberti et al. ${ }^{233}$ Here, we provide a detailed analysis of the matrix elements of the helical and linear cumulenes' Hamiltonian matrix $\mathbf{H}_{C}$, which may offer additional insights into the structural origin of the polarization. We will investigate why no polarization is observed for the linear molecule, and why the polarization for different helices changes. In addition to the structural origin of the polarization, we will investigate the relation between the imaginary part of the matrix elements of $\mathbf{H}_{C}$ in the scattering region and the spin-orbit coupling of the electrodes, comparing $\mathbf{H}_{C}$ within a gold-molecule-gold junction with $\mathbf{H}$ of the isolated molecule.

\subsubsection{Helicity dependence of imaginary Hamiltonian elements}

To investigate the importance of the helical structure for the polarization of the transmitted electrons, we take a closer look at the matrix elements of the isolated linear and helical molecules. We are focusing on a single nearest-neighbor $p$ - $p$ block of the $\mathbf{H}_{C}^{\uparrow \uparrow}$ block $\left(\mathbf{V}_{i,(i+1)}^{\uparrow \uparrow}\right.$; $i$ denotes an arbitrary atom in the molecule and $i+1$ the atom closest to atom $i$ along transport direction. $)_{5}^{5}$. For simplicity, we are using a modified basis set without polarization functions (i.e., no $p$-orbitals for hydrogen and no $d$-orbitals for carbon atoms; for details see

\footnotetext{
${ }^{5}$ Since the $s$-orbitals' contribution to the spin-orbit coupling is small, we will not discuss them in detail.
} 
Section A).
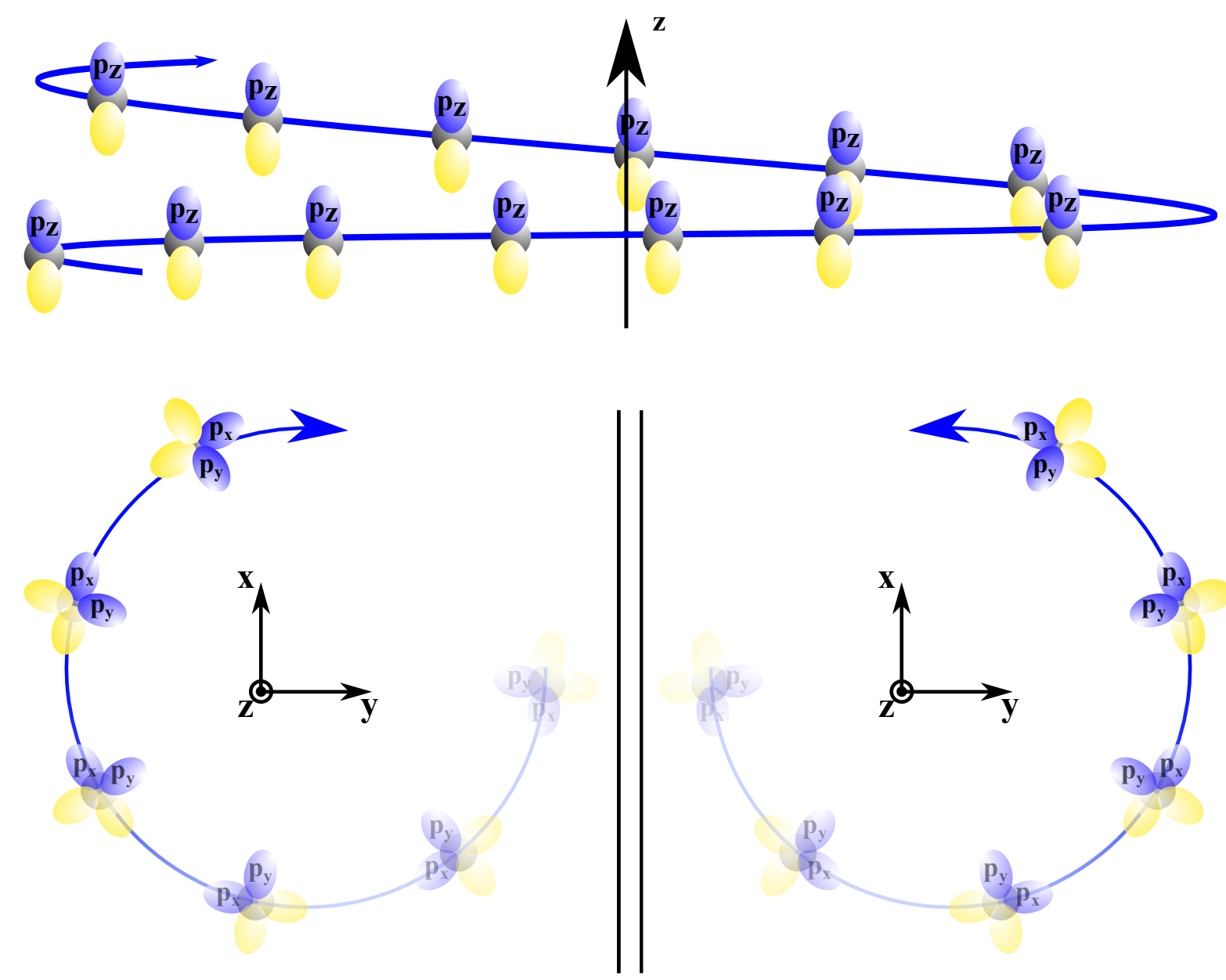

Figure 3: Orientation of the $p$ orbitals after having transformed the Cartesian basis into a helical basis. The $p_{x}$ orbitals are tangent to the helix and the positive lobe (blue) is pointing into ascending direction of the helix (indicated by the arrowhead). The $p_{z}$ orbitals remain oriented along the helix axis. The positive lobe of the $p_{y}$ orbitals is pointing into the center of the helix.

A problem in comparing the matrix elements of the left- and right-handed helical structure is the usage of a standard atom-centered basis set as provided by electronic structure codes, where the basis functions will be oriented along the Cartesian coordinate axes in a somewhat arbitrary fashion, even when aligning the molecules along a selected axis. In such a basis, the detailed values and signs of $\mathbf{S}$ and $\mathbf{H}_{C}$ comparing helices of different handedness will depend on the orientation of the molecule. To circumvent this problem, the basis is transformed into a helical basis, where the $p_{x}$ is tangent to the helix, and the positive lobe 
of the $p_{y}$ orbital points into the center of the helix. The positive lobe of the $p_{z}$ orbitals is oriented along the helix axis (see Figure 3; a similar scheme was used by Medina et al. $\frac{19}{}$ ).

First, we look at $\mathbf{V}_{i,(i+1)}^{\uparrow \uparrow}$ for the left- and right-handed helix. Upon changing the handedness of the helix, $\operatorname{Re}\left(\mathbf{V}_{i,(i+1)}^{\uparrow \uparrow}\right)$ does not change its sign, while $\operatorname{Im}\left(\mathbf{V}_{i,(i+1)}^{\uparrow \uparrow}\right)$ does (see Figure 4 ). This reinforces the importance of $\operatorname{Im}\left(\mathbf{H}_{C}\right)$ as the origin of the change in the polarization's sign upon changing the handedness of the helix.

\subsection{2 "Direction-dependence" of imaginary Hamiltonian elements}

While this may explain the different signs of the polarization for helices with different handedness, it does not give a simple picture of why the linear one does not show any polarization, since $\operatorname{Im}\left(\mathbf{H}_{C}\right)$ is also non-zero for the linear molecule. However, comparing $\operatorname{Im}\left(\mathbf{V}_{(i, i+1)}^{\uparrow \uparrow}\right)$ and $\operatorname{Im}\left(\mathbf{V}_{(i+1, i)}^{\uparrow \uparrow}\right)^{6}$ of the linear and helical molecule, a significant difference can be identified; For the helical molecule, $\operatorname{Im}\left(\mathbf{V}_{i,(i+1)}^{\uparrow \uparrow}\right)$ of the $p$-orbitals is different from $\operatorname{Im}\left(\mathbf{V}_{(i+1), i}^{\uparrow \uparrow}\right)$ of the p-orbitals, while for the linear molecule both are the same (see Figure 5). This indicates that the spin-orbit coupling induced $p-p$-nearest-neighbor coupling between the atoms in the perfect linear molecule is direction-independent, while for the perfect helical molecules, it is direction-dependent. An illustration of the origin of this difference is given in Figure 6 .

${ }^{6}$ This is based on interpreting the block $\left.\mathbf{V}_{i,(i+1}\right)$ as transition amplitudes against transport direction and the block $\left.\mathbf{V}_{(i+1}\right), i$ as transition amplitudes in transport direction, in line with considering such elements as nearest-neighbor hopping elements in tight-binding approaches. 


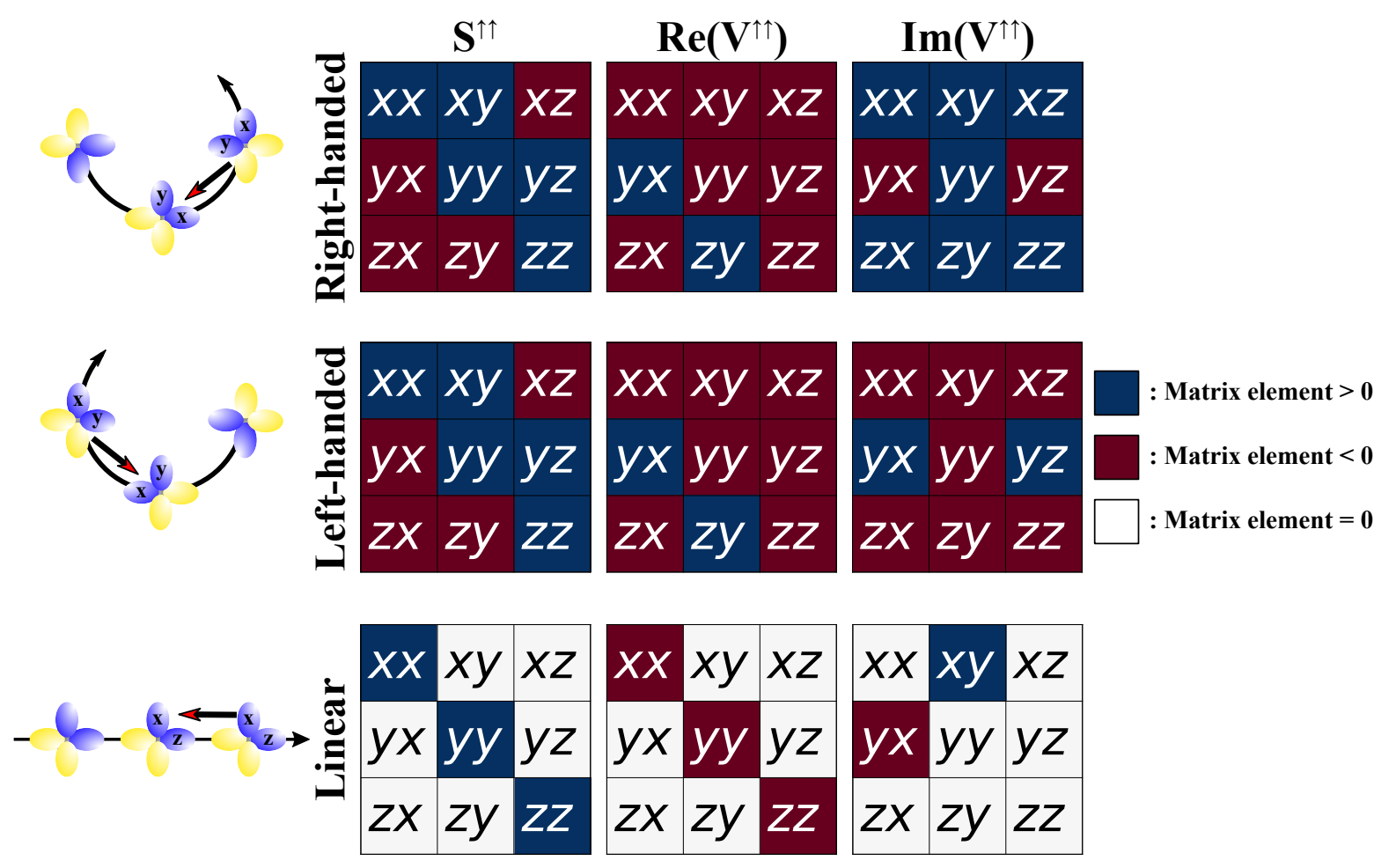

Figure 4: Exemplary block of $p-p$ orbital nearest-neighbor coupling in $\mathbf{H}_{C}^{\uparrow \uparrow}\left(\mathbf{V}_{i,(i+1)}^{\uparrow \uparrow}\right)$ in transport direction for the isolated molecules. Comparing the left- and right-handed helix in a helical basis, the elements of $\mathbf{S}_{i,(i+1)}^{\uparrow \uparrow}$ and $\operatorname{Re}\left(\mathbf{V}_{i,(i+1)}^{\uparrow \uparrow}\right)$ do not change their sign upon changing the handedness, all elements of $\operatorname{Im}\left(\mathbf{V}_{i,(i+1)}^{\uparrow \uparrow}\right)$ do so. This may be the origin of the change-of-sign in the polarization upon changing the handedness of the helix. For the linear molecule, much more elements are zero compared to the helical molecules. The plot was made for $\operatorname{Im}\left(\mathbf{H}^{\uparrow \uparrow}\right)$ for the isolated carbon helix, where non-zero matrix elements were defined to be larger than $10^{-8}$ a.u. (positive) and smaller than $-10^{-8}$ a.u. (negative). It has the same qualitative structure as $\operatorname{Im}\left(\mathbf{H}_{C}^{\uparrow \uparrow}\right)$ for the helix in the presence of gold electrodes (see Figure 7 for a comparison), but was chosen for its more regular structure. For simplicity, only one block of the $p-p$ coupling is shown (owing to the double-zeta basis, there are two such blocks, see Section A for details on the construction of the basis). 


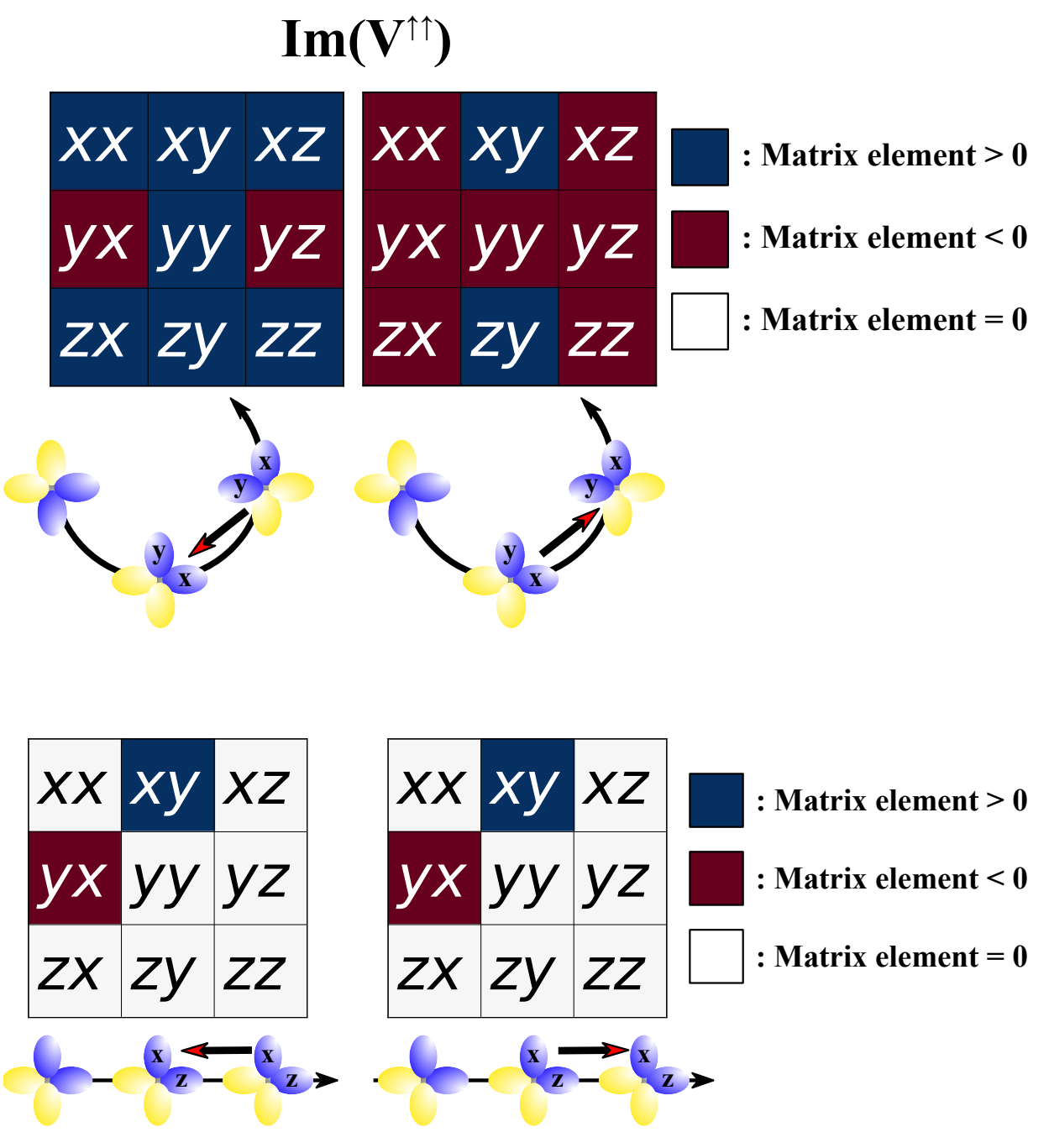

Figure 5: Exemplary block of $p-p$ orbital nearest-neighbor coupling in $\operatorname{Im}\left(\mathbf{H}_{C}^{\uparrow \uparrow}\right)\left(\operatorname{Im}\left(\mathbf{V}_{i,(i+1)}^{\uparrow \uparrow}\right)\right)$ along and against the transport direction, illustrating the Hermitian symmetry of the matrix. For the linear molecule, the sign of all elements in transport direction is the same as against transport direction, while for the right-handed helix, the sign of some elements in $\operatorname{Im}\left(\mathbf{V}_{i,(i+1)}^{\uparrow \uparrow}\right)$ changes comparing along and against transport direction. The same holds for the $\operatorname{Re}\left(\mathbf{V}_{i,(i+1)}^{\uparrow \uparrow}\right)$ of the $p$-orbitals. This implies that $T_{L R}^{\uparrow \uparrow}=T_{R L}^{\uparrow \uparrow}$ may be valid for the linear molecule, which would prevent any polarization of the transmitted electrons. The plot was made for $\operatorname{Im}\left(\mathbf{H}^{\uparrow \uparrow}\right)$ for the isolated carbon helix, where non-zero matrix elements were defined to be larger than $10^{-8}$ a.u. (positive) and smaller than $-10^{-8}$ a.u. (negative). It has the same qualitative structure as $\operatorname{Im}\left(\mathbf{H}_{C}^{\uparrow \uparrow}\right)$ for the helix in the presence of gold electrodes (see Figure 7 for a comparison), but was chosen for its more regular structure. For simplicity only one block of the $p-p$ coupling is shown (owing to the double-zeta basis, there are two such blocks, see Section A for details on the construction of the basis). 

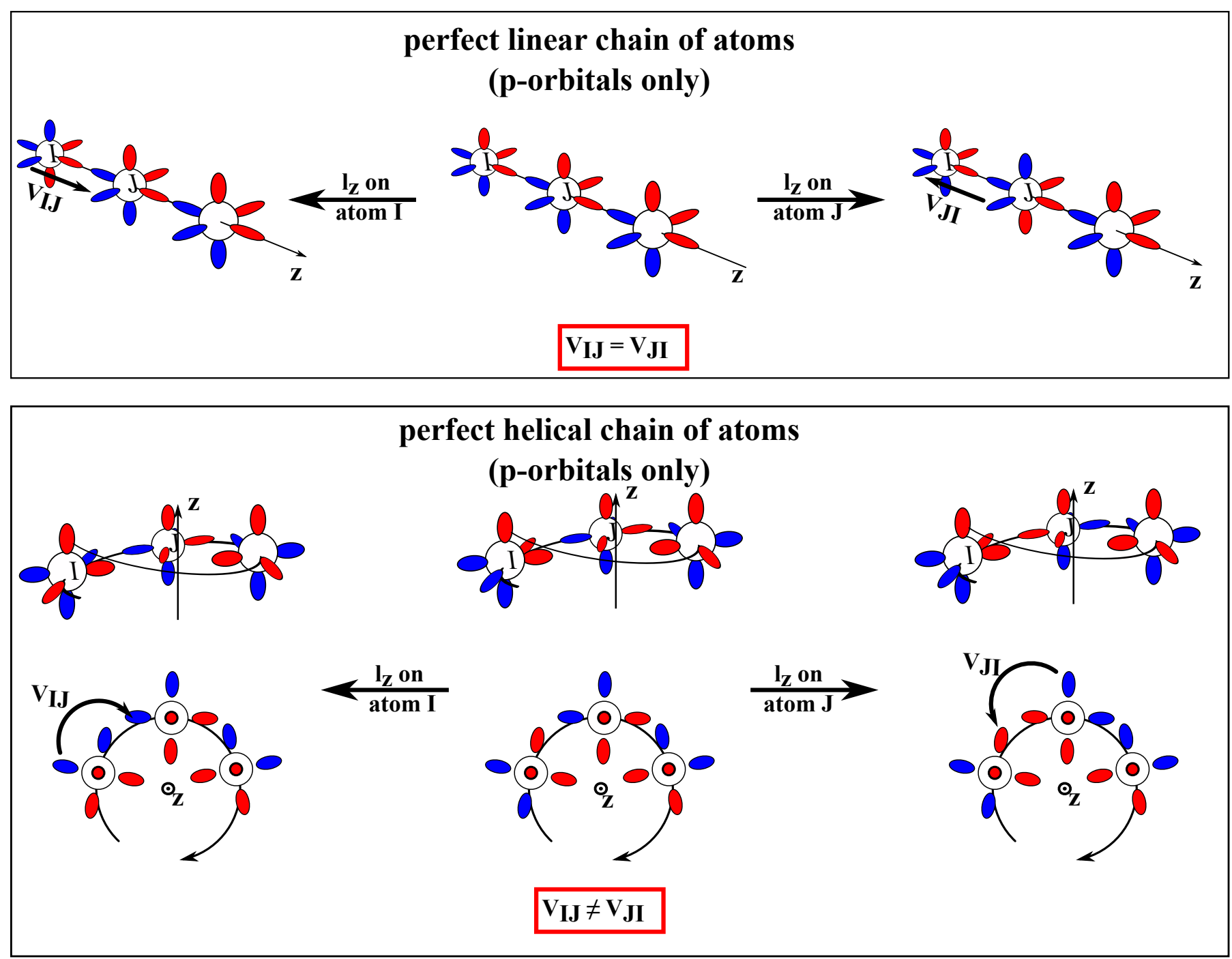

Figure 6: Direction dependence of nearest-neighbor spin-orbit matrix elements between $p$ atomic orbitals on atom $I$ and atom $J$, in and against transport direction ( $z$ axis). The size of such elements can be estimated by the overlap of a rotated $p$-orbital with an unrotated $p$-orbital (see Appendix B). For a perfect linear molecule, the $p-p$ overlap does not depend on which atom the orbitals were rotated on, while for the helical molecule in a helical basis, it does. The imaginary part of the $p-p$ coupling of atom $I$ to atom $J\left(\operatorname{Im}\left(\mathbf{V}_{I J}\right)\right)$ is different from the coupling of atom $J$ to atom $I\left(\operatorname{Im}\left(\mathbf{V}_{J I}\right)\right)$, indicating a possible difference of the transport in and against transport direction.

This may be the reason for the absence of polarization in the linear molecule, since the direction-independent imaginary $p-p$ coupling may indicate that $T_{R L}^{\uparrow \uparrow}=T_{L R}^{\uparrow \uparrow}$. In combination with our derived relation $T_{R L}^{\uparrow \uparrow}=T_{L R}^{\downarrow \downarrow}$, we retain the relation $T_{R L}^{\uparrow \uparrow}=T_{L R}^{\downarrow \downarrow}=T_{R L}^{\uparrow \uparrow}=T_{L R}^{\downarrow \downarrow}$ from the SOC-free case, which prohibits any polarization. The causality of the relation 
between direction-independent coupling and absence of spin polarization (and its generality) have to be verified in future work. It is important to note that direction-independent coupling is also present for the $p-p$ and $s-s$ blocks of $\operatorname{Re}\left(\mathbf{V}_{i,(i+1)}^{\uparrow \uparrow}\right) / \operatorname{Re}\left(\mathbf{V}_{(i+1), i}^{\uparrow \uparrow}\right)$, and the $s-p$ blocks of $\operatorname{Im}\left(\mathbf{V}_{i,(i+1)}^{\uparrow \uparrow}\right) / \operatorname{Im}\left(\mathbf{V}_{(i+1), i}^{\uparrow \uparrow}\right)$. However, it is not the case for the $s-p$ block of $\operatorname{Re}\left(\mathbf{V}_{i,(i+1)}^{\uparrow \uparrow}\right) / \operatorname{Re}\left(\mathbf{V}_{(i+1), i}^{\uparrow \uparrow}\right)$ (owing to the "direction-dependence" of overlap between $s$ and $p$ orbitals, see Supporting Information for the whole matrices). Nevertheless, we assume $\operatorname{Im}\left(\mathbf{H}_{C}^{\uparrow}\right)$ to be most important for the CISS effect, because this part originates from the spin-orbit coupling and is a prerequisite for non-zero polarization.

\subsubsection{A proximity effect of gold?}

To investigate the influence of the gold electrodes on the effective spin-orbit coupling of the molecules, we look at $\mathbf{H}_{C}$ of the gold-molecule-gold junction and compare it with the Hamiltonian matrix $\mathbf{H}$ of the isolated helical molecule (see Figure 7). The proximity of the electrodes induces a massive increase of $\operatorname{Im}\left(\mathbf{H}_{C}\right)$ as well as of $\operatorname{Re}\left(\mathbf{H}_{C}^{\uparrow \downarrow}\right) / \operatorname{Re}\left(\mathbf{H}_{C}^{\downarrow \uparrow}\right)$ and leads to less block-diagonal shape of the matrix. This implies spin-orbit coupling becoming less local due to the vicinity of the electrodes. The spin-orbit coupling in our calculations, therefore, is partially inherited from the gold electrodes in our model systems, similar to known proximity effects in graphene on gold ${ }^{50151}$ (also compare Ref. ${ }^{[52}$ ). 


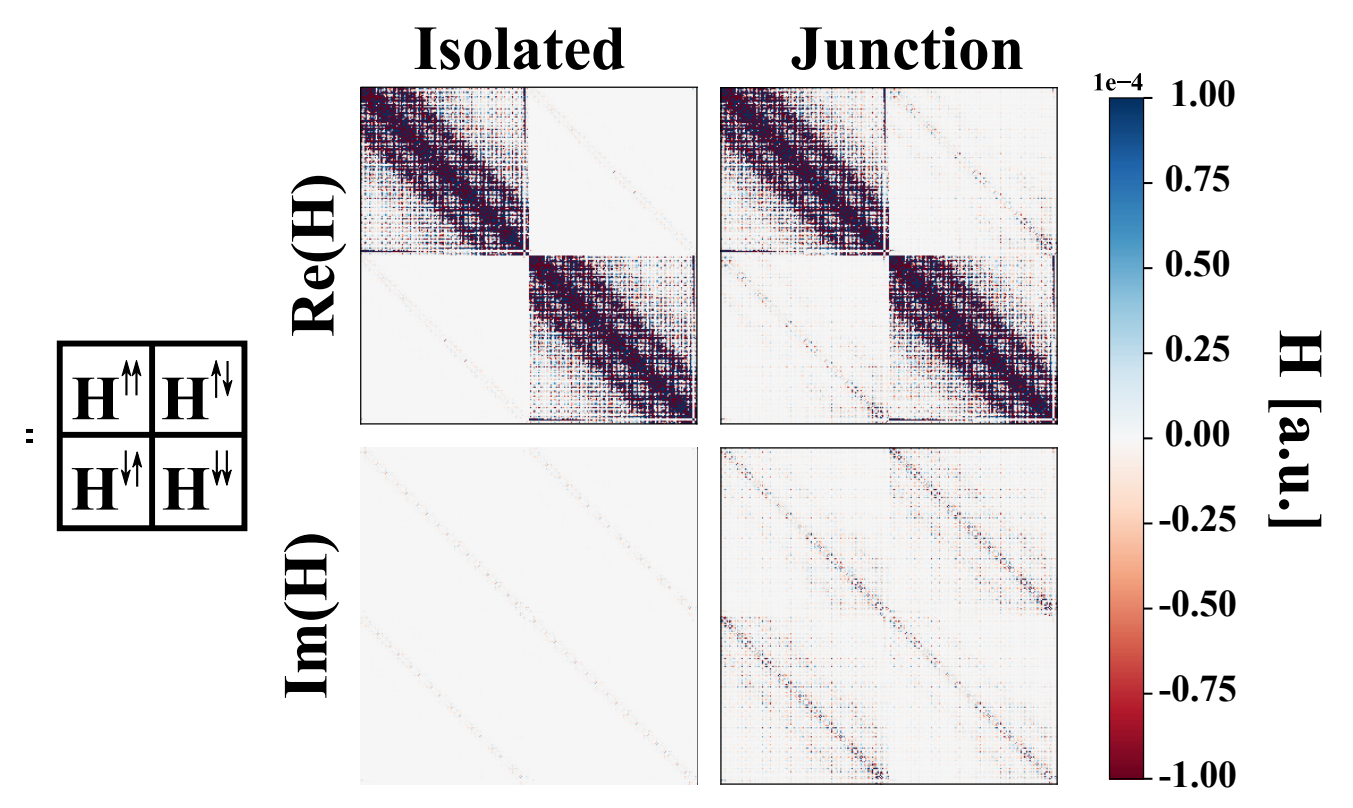

Structure Hamiltonian matrix

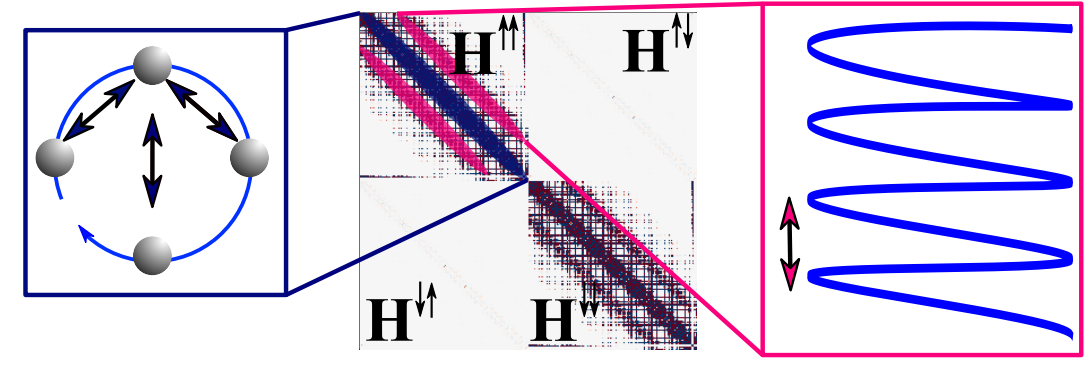

Figure 7: a): $\operatorname{Re}\left(\mathbf{H}_{(C)}\right)$ (upper part) and $\operatorname{Im}\left(\mathbf{H}_{(C)}\right.$ ) (lower part) for the carbon atoms of the right-handed helical cumulene only. On the left-hand side the matrices are depicted for the isolated molecule, on the right-hand side they are depicted for the gold-moleculegold junction. The gold electrodes induces a massive increase of $\operatorname{Im}\left(\mathbf{H}_{C}\right), \operatorname{Re}\left(\mathbf{H}_{C}^{\uparrow \downarrow}\right)$ and $\operatorname{Re}\left(\mathbf{H}_{C}^{\downarrow \uparrow}\right)$ within the helix. b): Schematic overview of the structure of the Hamiltonian matrix. While the main diagonal (marked in blue) arises from the interaction of the closest carbon atoms within a convolution, the off-diagonals arise from the interaction of carbon atoms of different convolutions.

The apparent proximity effect in our calculations contrasts with experimental results on photoemission of chiral molecules on aluminium and copper surfaces, $\frac{1114}{14}$ which demonstrate that substrate SOC is not required for CISS in that type of experiment. Therefore, further work will be necessary to decide whether our computationally observed proximity effect is a general feature of realistic molecules in metal junctions, or whether it is particular to the simplified carbon structure and the way it is studied here, both from the theoretical and 
the experimental side. In particular, it might be instructive to replace gold electrodes (as employed in Ref. ${ }^{12}$ ) by copper ones in single-molecule transport experiments: It cannot be excluded that the proven independence of CISS on substrate SOC in photoemission is not directly transferable to electron transport, since in the former case unbound and in the latter (quasi-)bound single-particle states are involved.

\section{Lessons from a barrier model}

A related insight connecting spin-orbit coupling and imaginary quantities has also recently been gained in the context of barrier scattering. A barrier model neglects all aspects of electron correlation. Despite this drastic simplification, substantial physical insight can be obtained from this simple system: In a recent publication Varela et al. have carried out a detailed study of a tunneling process including spin-orbit coupling. ${ }^{53}$ The remarkable fact, which had been completely ignored in the literature, is that the inclusion of SOC translates into an imaginary component of the $k$-vector in the barrier region, thereby strongly modifying the length dependence of tunneling-mediated electron transfer. A highly consistent scenario arises where two concomitant mechanisms for spin selection arise through spin precession and differential spin amplitude decay. High spin filtering can take place at the cost of reduced

amplitude transmission, while angular momentum conservation is preserved. ${ }^{53}$ This finding is important because it immediately relates to our own results that it is the imaginary part of the effective single-particle Hamiltonian matrix, in turn determined by SOC, that allows non-vanishing spin polarization. 


\section{Connection between spin polarization in barrier scat- tering and transmission}

The description of the CISS effect within a barrier model can be recast in a way that the connection with transmission is made apparent. We discuss this connection for the coherent case where thermal and inelastic scattering are not explicitly included. The starting point is the connection between the density matrix of a system $\boldsymbol{\rho}$ and the spin polarization, 18

$$
P_{\mu}=\frac{\operatorname{Tr}\left[\boldsymbol{\sigma}_{\mu} \boldsymbol{\rho}\right]}{\operatorname{Tr}[\boldsymbol{\rho}]} .
$$

$\mu$ denotes a single spacial component of the polarization $(x, y$ and $z)$ and $\boldsymbol{\sigma}_{\mu}$ denotes the corresponding Pauli spin matrix. We are interested in the polarization of the outcoming electrons (electrons which were scattered through the barrier). The density matrix of the outcoming electrons $\boldsymbol{\rho}^{\text {out }}$ is connected to the density matrix of the incoming electrons $\boldsymbol{\rho}^{\text {out }}$ $\operatorname{as}^{54}$

$$
\rho^{\text {out }}=\mathbf{f}^{\dagger} \boldsymbol{\rho}^{\text {in }} \mathbf{f}
$$

where $\mathbf{f}$ is the scattering matrix. Using Equation (17) the polarization of the outcoming electrons can be written as

$$
P_{\mu}^{\text {out }}=\frac{\operatorname{Tr}\left(\boldsymbol{\sigma}_{\mu} \mathbf{f}^{\dagger} \rho^{i n} \mathbf{f}\right)}{\operatorname{Tr}\left(\mathbf{f}^{\dagger} \rho^{i n} \mathbf{f}\right)} .
$$

In connection to the calculations done in this work, we are interested in $P_{z}$ (which we have simply referred to as $P$ above) for unpolarized incoming electrons with a density matrix

$$
\boldsymbol{\rho}^{i n}=\frac{1}{2}\left[\begin{array}{ll}
1 & 0 \\
0 & 1
\end{array}\right] \text {. }
$$


Inserting Equation (20) and the definition of $\boldsymbol{\sigma}_{z}$

$$
\boldsymbol{\sigma}_{\boldsymbol{z}}=\left[\begin{array}{cc}
1 & 0 \\
0 & -1
\end{array}\right]
$$

into Equation $(19)$, we obtain

$$
P_{z}^{\text {out }}=\frac{\operatorname{Tr}\left(\boldsymbol{\sigma}_{z} \mathbf{f}^{\dagger}\left[\begin{array}{ll}
1 & 0 \\
0 & 1
\end{array}\right] \mathbf{f}\right)}{\operatorname{Tr}\left(\mathbf{f}^{\dagger}\left[\begin{array}{ll}
1 & 0 \\
0 & 1
\end{array}\right] \mathbf{f}\right)}=\frac{\operatorname{Tr}\left(\boldsymbol{\sigma}_{z} \mathbf{f}^{\dagger} \mathbf{t}\right)}{\operatorname{Tr}\left(\mathbf{t}^{\dagger} \mathbf{t}\right)}
$$

for the polarization in $z$-direction. For simplicity, we now define an auxiliary matrix

$$
\Phi=\mathbf{f}^{\dagger} \mathbf{f}=\left[\begin{array}{ll}
\Phi_{\uparrow \uparrow} & \Phi_{\uparrow \downarrow} \\
\Phi_{\downarrow \uparrow} & \Phi_{\downarrow \downarrow}
\end{array}\right]
$$

where we only define the diagonal blocks, because later only the trace of this matrix is needed, as 18

$$
\begin{aligned}
& \Phi_{\uparrow \uparrow}=\left(\frac{m}{2 \pi \hbar^{2}}\right)^{2}\left(\left|t_{\uparrow \uparrow}\right|^{2}+\left|t_{\downarrow \uparrow}\right|^{2}\right) \\
& \Phi_{\downarrow \downarrow}=\left(\frac{m}{2 \pi \hbar^{2}}\right)^{2}\left(\left|t_{\downarrow \downarrow}\right|^{2}+\left|t_{\uparrow \downarrow}\right|^{2}\right) .
\end{aligned}
$$

These diagonal elements are spin-depended blocks of the t-matrix and correspond physically to the total transmission associated with each spin channel for spin-1/2 particles. If we insert 
Equation (24) into Equation (19) we get

$$
P_{z}^{\text {out }}=\frac{\operatorname{Tr}\left(\boldsymbol{\sigma}_{z} \boldsymbol{\Phi}\right)}{\operatorname{Tr}(\boldsymbol{\Phi})}=\frac{\operatorname{Tr}\left[\begin{array}{cc}
\Phi_{\uparrow \uparrow} & \Phi_{\uparrow \downarrow} \\
-\Phi_{\downarrow \uparrow} & -\Phi_{\downarrow \downarrow}
\end{array}\right]}{\operatorname{Tr}\left[\begin{array}{cc}
\Phi_{\uparrow \uparrow} & \Phi_{\uparrow \downarrow} \\
\Phi_{\downarrow \uparrow} & \Phi_{\downarrow \downarrow}
\end{array}\right]}=\frac{\left(\Phi_{\uparrow \uparrow}-\Phi_{\downarrow \downarrow}\right)}{\left(\Phi_{\uparrow \uparrow}+\Phi_{\downarrow \downarrow}\right)}
$$

which we can also write as

$$
P_{z}^{\text {out }}=\frac{\left(\left|t_{\uparrow \uparrow}\right|^{2}+\left|t_{\downarrow \uparrow}\right|^{2}-\left|t_{\downarrow \downarrow}\right|^{2}-\left|t_{\uparrow \downarrow}\right|^{2}\right)}{\left(\left|t_{\uparrow \uparrow}\right|^{2}+\left|t_{\downarrow \uparrow}\right|^{2}+\left|t_{\downarrow \downarrow}\right|^{2}+\left|t_{\uparrow \downarrow}\right|^{2}\right)}
$$

which is proportional to the polarization defined above in a transmission language. This suggests that the findings on spin-orbit coupling leading to imaginary $k$ vectors and reduced decay under the barrier described in Section 4 and on spin-orbit coupling leading to imaginary parts in the Hamiltonian matrix studied in the first parts of this manuscript may be related.

\section{Conclusion and Outlook}

Chiral-induced spin selectivity is an intriguing phenomenon, observed in a variety of helical light-atom molecules. So far, it has been well established that spin-orbit coupling is essential for CISS, but the observed magnitudes of the effect remain puzzling. To provide insight into what is missing in present-day theoretical descriptions, we analyze analytically how the imaginary parts of the effective single-particle Hamiltonian matrix $\mathbf{H}_{C}$ induced by spinorbit coupling, combined with the symmetric imaginary self-energies, lead to nonsymmetric Green's functions and how this can result in spin polarization.

Based on first-principles Kohn-Sham DFT calculations, we show how these imaginary parts change sign upon changing the helix sense, thus providing a direct link with the helicity- 
dependent spin polarization of transmitted electrons in helical structures. Importantly, the presence of imaginary parts in $\mathbf{H}_{C}$ alone does not automatically lead to polarization, as exemplified by the fact that also the linear carbon chain has imaginary elements in $\mathbf{H}_{C}$. However, when comparing the "hopping-type" elements in and against transport direction for the helix of a given helix sense, we find that the imaginary elements which change sign in the helix are the ones which are zero in the linear chain. Conversely, when all imaginary terms are deleted in the Hamiltonian of a helical structure, zero polarization results, reinforcing the importance of these terms for CISS.

We show that the imaginary part of $\mathbf{H}_{C}$ (and thus polarization) is strongly increased by the presence of gold atoms to which the carbon helix couples, similar to the known proximity effect in graphene. This could indicate that in contrast to photoemission experiments, where CISS has been observed on substrates with weak spin-orbit coupling, in electron transport measurements the substrate spin-orbit coupling does play an important role. This would be an interesting direction for experiment, e.g. by repeating the single-molecule break junction experiments discussed in Ref. ${ }^{12}$ with copper rather than gold electrodes. On the other hand, our calculations point out the need for further studies on such proximity effects and their actual relevance for a first-principles description of CISS. Such work is currently under way in our laboratories. More generally, the consequences of chirality for the molecule-metal interface may have to be taken into account more explicitly in first-principles simulations, as suggested by recent theoretical ${ }^{26}$ and experimental work $\frac{1155}{}$.

The importance of the imaginary part of the Hamiltonian matrix for spin polarization has an important consequence for modeling CISS with present-day Kohn-Sham DFT: Since the imaginary part can not only originate from spin-orbit coupling, but also from exactexchange admixture in the exchange-correlation functional, $\frac{47}{4}$ the choice of this functional could have an important effect on spin polarization. This will be the subject of follow-up work.

Dephasing has been discussed as essential for CISS in the context of reduced tight-binding 
models, $24 \sqrt[25]{2}$ and having spin polarization only when adding local leakages is a feature of many simplified models. $\stackrel{56}{56}$ Our results demonstrate that a first-principles approach allows for spin polarisation in the absence of such local leakages and dephasing terms. Yet, the relevance of imaginary terms demonstrated here could reinforce the lesson learned from these simpler approaches, that dephasing (resulting, e.g., from interactions with vibrational modes or with the molecule's environment) or leakage to the environment could enhance spin polarization and could therefore be important missing building blocks for our first-principles theories of CISS: When modeling such processes in terms of Büttiker probes, $\frac{57-62}{62}$ imaginary terms arise. In the Landauer approach employed here, these terms are not considered (except for leakage into the two-terminal electrodes). Including such terms explicitly might lead towards a first-principles theory able to account for the surprisingly large CISS in helical molecules.

Finally, we draw an analogy between polarization in electron transmission and in barrier scattering, where it has recently been shown that spin-orbit coupling leads to imaginary $k$ vectors, reducing the decay of the wave function under the barrier. This could imply that analyzing the imaginary components involved in helical structures in the presence of spinorbit coupling could help understand the effective electron transfer in biological systems over long distances.

\section{Acknowledgments}

We would like to thank Rafael Gutierrez and Sibylle Gemming for helpful discussions and the high-performance computing centre at University of Hamburg for computational resources. This work is supported by Deutsche Forschungsgemeinschaft (DFG) via the project "Structure-property relationships for spin-orbit effects in chiral molecules" (HE 5675/4-1). 


\section{A Computational methods}

The (Constrained) structure optimizations of the hydrogen atoms in our ideal helical and linear molecules were carried out using TURBOMOLE 7.1. $\frac{63}{66}$ The exchange-correlation functional introduced by Perdew, Burke and Ernzerhof $(\mathrm{PBE})^{[67 / 68}$ and the def2-TZVP $\frac{69 / 70}{b a s i s}$ set were employed. Grimme's dispersion correction DFT-D3 ${ }^{71}$ including Becke-Johnson damping ${ }^{72}$ was used. To speed up the structure optimizations, the resolution-of-the-identity method $\sqrt{73 \sqrt{74}}$ with the corresponding auxiliary basis set ${ }^{75}$ was applied. The hydrogen atoms were allowed to relax until the change of energy was below $10^{-6}$ a.u. and the gradient was below $10^{-4}$ a.u.. The energy convergence threshold within the self-consistent field algorithm was set to $10^{-7}$ a.u..

The optimized structures were used to build the electrode-molecule-electrode structures by simply placing the optimized isolated structure between two 10-atomic gold clusters. Based on these electrode-molecule-electrode structures one-component and a twocomponent KS-DFT single-point calculations ${ }^{46 / 76}$ were carried out using TURBOmOLE 7.1. If not mentioned otherwise the B3LYP $\stackrel{77-79}{79}$ hybrid functional was used. Scalar-relativistic effects and spin-orbit coupling were considered within the exact two-component (X2C) method $\stackrel{80181}{ }$ using the corresponding $x 2 c-S V P a l l-2 c^{82}$ basis set for all atoms. If mentioned, a modified version of this basis set, removing the polarization functions for the hydrogen and carbon atoms was used.

Every junction was, at first, calculated using a one-component KS-DFT calculation considering scalar-relativistic effects only. The converged molecular orbitals were then used as an initial guess for the subsequent two-component KS-DFT calculation, including spin-orbit coupling. The Hamiltonian matrix of the scalar-relativistic calculation was then used to build the electrodes' self-energies, while the Hamiltonian matrix of the spin-orbit coupling DFT calculation was used to build the Hamiltonian matrix of the central region. 
To build the helical basis for the detailed matrix analysis (see Figure 3) we removed the $p$-orbitals for the hydrogen (remaining basis functions: two $s$-orbitals) and the $d$-orbitals for the carbon atoms (remaining basis functions: three $s$-orbitals and two $p$-orbitals). Having done the DFT calculation we then did a unitary transformation of the Fock- and overlap matrix corresponding to a transformation of any atom's local-axis system. $\stackrel{83}{73}$ The local $x$-axes were rotated onto the connection line between the two nearest-neighbor atoms (Around the $z$-axis). The local $y$-axis of the resulting local axis system, if necessary, was then transformed with a mirror operation at the $x z$-plane, so that the $y$-axis points into the center of the helix. The resulting $p_{x^{-}}$and $p_{y}$-orbitals (named in the local axis system) remain in the global $x y$ plane while the local $z$-axes remain oriented along the helix axis.

\section{B Spin-orbit coupling operator and matrix elements}

Spin-orbit coupling as an origin of imaginary parts in the Hamiltonian matrix can be shown by formulating the $p-p$ matrix elements of a single electron spin-orbit coupling operator as ${ }^{84}$

$$
\left\langle p_{i}\left|\hat{H}_{\mathrm{SOC}}\right| p_{j}\right\rangle=\xi_{k}\left\langle p_{i}|\hat{\mathbf{1}} \cdot \hat{\mathbf{s}}| p_{j}\right\rangle,
$$

where $\xi_{k}$ is a single electron spin-orbit coupling constant. The angular-momentum operator

$\hat{\mathbf{l}}$ is a vector $\left(\hat{l}_{x}, \hat{l}_{y}, \hat{l}_{z}\right)$ as well as the spin-momentum operator $\hat{\mathbf{s}}\left(\hat{s}_{x}, \hat{s}_{y}, \hat{s}_{z}\right)$. Taking a closer look at $\hat{\mathbf{l}}$, its $y$ and $x$ components are typically written using the shift operators $\hat{l}_{+}$ and $\hat{l}_{-} \underline{84}$

$$
\begin{aligned}
& \hat{l}_{y}=\frac{-\mathrm{i}}{2}\left(\hat{l}_{+}-\hat{l}_{-}\right) \\
& \hat{l}_{x}=\frac{1}{2}\left(\hat{l}_{+}+\hat{l}_{-}\right)
\end{aligned}
$$

These operators act on orbitals/wave functions defined by the quantum number $l$ (angular momentum) and $m_{j}$ (angular-momentum projected along the $z$ axis). These orbitals/wave 
function are then often written as $\left|l, m_{l}\right\rangle$. The action of the operators on those functions are defined as ${ }^{84}$

$$
\begin{aligned}
& \hat{l}_{z}\left|l, m_{l}\right\rangle=m_{l}\left|l, m_{l}\right\rangle \\
& \hat{l}_{+}\left|l, m_{l}\right\rangle=\sqrt{\left(l+m_{l}+1\right)\left(l-m_{l}\right)}\left|l, m_{l}+1\right\rangle \\
& \hat{l}_{-}\left|l, m_{l}\right\rangle=\sqrt{\left(l-m_{l}+1\right)\left(l+m_{l}\right)}\left|l, m_{l}-1\right\rangle .
\end{aligned}
$$

The typically used $p$ orbitals $\left(p_{x}, p_{y}, p_{z}\right)$ are superpositions of such functions ${ }^{85}$ and can be defined as

$$
\begin{aligned}
& \left|p_{z}\right\rangle=\left|p_{0}\right\rangle \\
& \left|p_{y}\right\rangle=\frac{i}{\sqrt{2}}\left(\left|p_{-1}\right\rangle+\left|p_{+1}\right\rangle\right) \\
& \left|p_{x}\right\rangle=\frac{1}{\sqrt{2}}\left(\left|p_{-1}\right\rangle-\left|p_{+1}\right\rangle\right),
\end{aligned}
$$

where $\left|p_{0}\right\rangle$ is the $p$ orbital with $m_{l}=0,\left|p_{+1}\right\rangle$ the $p$ orbital with $m_{l}=1$, and $\left|p_{-1}\right\rangle$ the $p$ orbital with $m_{l}=-1$. The effect of the $\hat{\mathbf{L}}$ operator on the $p_{z}$ orbital therefore is

$$
\begin{aligned}
& \hat{l}_{z}\left|p_{z}\right\rangle=0 \\
& \hat{l}_{y}\left|p_{z}\right\rangle=\frac{-i \sqrt{2}}{2}\left(\left|p_{+1}\right\rangle-\left|p_{-1}\right\rangle\right)=i\left|p_{x}\right\rangle \\
& \hat{l}_{x}\left|p_{z}\right\rangle=\frac{\sqrt{2}}{2}\left(\left|p_{+1}\right\rangle+\left|p_{-1}\right\rangle\right)=-i\left|p_{y}\right\rangle,
\end{aligned}
$$


thus rotating the $p_{z}$ orbital onto the $p_{y} / p_{x}$ orbital, depending on which part of the vector is considered. The effect on the $p_{y}$ orbital

$$
\begin{aligned}
& \hat{l}_{z}\left|p_{y}\right\rangle=\frac{i}{\sqrt{2}}\left(\left|p_{+1}\right\rangle-\left|p_{-1}\right\rangle\right)=-i\left|p_{x}\right\rangle \\
& \hat{l}_{y}\left|p_{y}\right\rangle=0 \\
& \hat{l}_{x}\left|p_{y}\right\rangle=\frac{-i}{2}\left(\left|p_{0}\right\rangle+\left|p_{0}\right\rangle\right)=i\left|p_{z}\right\rangle
\end{aligned}
$$

and the $p_{x}$ orbital

$$
\begin{aligned}
& \hat{l}_{z}\left|p_{x}\right\rangle=\frac{1}{\sqrt{2}}\left(-\left|p_{-1}\right\rangle-\left|p_{+1}\right\rangle\right)=i\left|p_{y}\right\rangle \\
& \hat{l}_{y}\left|p_{x}\right\rangle=\frac{i}{2}\left(\left|p_{0}\right\rangle+\left|p_{0}\right\rangle\right)=-i\left|p_{z}\right\rangle \\
& \hat{l}_{x}\left|p_{x}\right\rangle=0
\end{aligned}
$$

give a similar relation, by rotating the $p$ orbitals along a specific axis onto another $p$ orbital. In addition to the $\hat{\mathbf{L}}$ operator the $\hat{\mathbf{s}}$ has to be considered, looking at SOC. Again the $y$ and $x$ components are typically written using the shift operators $\hat{s}_{+}$and $\hat{s}_{-} 84$

$$
\begin{aligned}
& \hat{s}_{y}=\frac{-\mathrm{i}}{2}\left(\hat{s}_{+}-\hat{s}_{-}\right) \\
& \hat{s}_{x}=\frac{1}{2}\left(\hat{s}_{+}+\hat{s}_{-}\right) .
\end{aligned}
$$

The orbitals/wave function on which these operator act on are typically defined by the quantum number $S$ (total spin) and $M_{S}$ (projection of the spin onto the $z$ axis) and are written as $\left|s, m_{s}\right\rangle$. The components of the $\hat{\mathbf{s}}$ operator are acting on the orbital/wave function 
$\operatorname{as}^{84}$

$$
\begin{aligned}
& \hat{s}_{z}\left|s, m_{s}\right\rangle=m_{s}\left|s, m_{s}\right\rangle \\
& \hat{s}_{+}\left|s, m_{s}\right\rangle=\sqrt{\left(s+m_{s}+1\right)\left(s-m_{s}\right)}\left|s, m_{s}+1\right\rangle \\
& \hat{s}_{-}\left|s, m_{s}\right\rangle=\sqrt{\left(s-m_{s}+1\right)\left(s+m_{s}\right)}\left|s, m_{s}-1\right\rangle .
\end{aligned}
$$

If we now again look at the $p$ orbitals ( $p_{z}$ as an example) with a spin quantum number $\left(S=\frac{1}{2}, m_{s}=\frac{1}{2}(\uparrow)\right.$ and $\left.m_{s}=-\frac{1}{2}(\downarrow)\right)$ the effect on the $\left|p_{z}^{\uparrow}\right\rangle$ is

$$
\begin{aligned}
\hat{s}_{z}\left|p_{z}^{\uparrow}\right\rangle & =\frac{1}{2}\left|p_{z}^{\uparrow}\right\rangle \\
\hat{s}_{y}\left|p_{z}^{\uparrow}\right\rangle & =\frac{i}{2}\left|p_{z}^{\downarrow}\right\rangle \\
\hat{s}_{x}\left|p_{z}^{\uparrow}\right\rangle & =\frac{1}{2}\left|p_{z}^{\downarrow}\right\rangle
\end{aligned}
$$

and on the $\left|p_{z}^{\downarrow}\right\rangle$

$$
\begin{aligned}
\hat{s}_{z}\left|p_{z}^{\downarrow}\right\rangle & =-\frac{1}{2}\left|p_{z}^{\downarrow}\right\rangle \\
\hat{s}_{y}\left|p_{z}^{\downarrow}\right\rangle & =-\frac{i}{2}\left|p_{z}^{\uparrow}\right\rangle \\
\hat{s}_{x}\left|p_{z}^{\downarrow}\right\rangle & =\frac{1}{2}\left|p_{z}^{\uparrow}\right\rangle
\end{aligned}
$$

leading to a spin-conserving effect of the $\hat{s}_{z}$ operator and spin-flip effect of the $\hat{s}_{y}$ and $\hat{s}_{x}$ operators. If both the $\hat{\mathbf{l}}$ and $\hat{\mathbf{s}}$ operator are combined

$$
\lambda(\hat{\mathbf{l}} \cdot \hat{\mathbf{s}})=\lambda\left(\hat{l}_{x} \hat{s}_{x}+\hat{l}_{y} \hat{s}_{y}+\hat{l}_{z} \hat{s}_{z}\right)
$$


the non-zero spin-orbit coupling elements between the $p$ orbitals are

$$
\begin{gathered}
\left\langle p_{y}^{\uparrow}\left|\hat{l}_{x} \hat{s}_{x}\right| p_{z}^{\downarrow}\right\rangle=\left\langle p_{y}^{\downarrow}\left|\hat{l}_{x} \hat{s}_{x}\right| p_{z}^{\uparrow}\right\rangle=-\left\langle p_{z}^{\downarrow}\left|\hat{l}_{x} \hat{s}_{x}\right| p_{z}^{\uparrow}\right\rangle=-\left\langle p_{z}^{\uparrow}\left|\hat{l}_{x} \hat{s}_{x}\right| p_{y}^{\downarrow}\right\rangle=\frac{-i}{2} \\
\left\langle p_{x}^{\uparrow}\left|\hat{l}_{y} \hat{s}_{y}\right| p_{z}^{\downarrow}\right\rangle=-\left\langle p_{x}^{\downarrow}\left|\hat{l}_{y} \hat{s}_{y}\right| p_{z}^{\uparrow}\right\rangle=\left\langle p_{z}^{\downarrow}\left|\hat{l}_{y} \hat{s}_{y}\right| p_{x}^{\uparrow}\right\rangle=-\left\langle p_{z}^{\uparrow}\left|\hat{l}_{y} \hat{s}_{y}\right| p_{z}^{\downarrow}\right\rangle=\frac{1}{2} \\
\left\langle p_{x}^{\uparrow}\left|\hat{l}_{z} \hat{s}_{z}\right| p_{y}^{\uparrow}\right\rangle=-\left\langle p_{x}^{\downarrow}\left|\hat{l}_{z} \hat{s}_{z}\right| p_{y}^{\downarrow}\right\rangle=\left\langle p_{y}^{\downarrow}\left|\hat{l}_{z} \hat{s}_{z}\right| p_{x}^{\downarrow}\right\rangle=-\left\langle p_{y}^{\uparrow}\left|\hat{l}_{z} \hat{s}_{z}\right| p_{x}^{\uparrow}\right\rangle=\frac{-i}{2}
\end{gathered}
$$

since $\left\langle p_{i}^{\sigma} \mid p_{j}^{\sigma^{\prime}}\right\rangle$ is zero if $i \neq j$ and $\sigma \neq \sigma^{\prime}$ when both orbitals are located on the same atom.

\section{Details on the calculation of the transmission func- tion}

We calculate the spin-dependent transmission functions as ${ }^{38}$

$$
\begin{aligned}
& T_{R L}^{\uparrow \uparrow}=\operatorname{Tr}\left[\boldsymbol{\Gamma}_{L}^{\uparrow \uparrow} \mathbf{G}^{\uparrow \uparrow} \boldsymbol{\Gamma}_{R}^{\uparrow \uparrow}\left(\mathbf{G}^{\uparrow \uparrow}\right)^{\dagger}\right] \\
& T_{R L}^{\downarrow \uparrow}=\operatorname{Tr}\left[\boldsymbol{\Gamma}_{L}^{\uparrow \uparrow} \mathbf{G}^{\downarrow \uparrow} \boldsymbol{\Gamma}_{R}^{\downarrow \downarrow}\left(\mathbf{G}^{\downarrow \uparrow}\right)^{\dagger}\right] \\
& T_{R L}^{\uparrow \downarrow}=\operatorname{Tr}\left[\boldsymbol{\Gamma}_{L}^{\downarrow \downarrow} \mathbf{G}^{\uparrow \downarrow} \boldsymbol{\Gamma}_{R}^{\uparrow \uparrow}\left(\mathbf{G}^{\uparrow \downarrow}\right)^{\dagger}\right] \\
& T_{R L}^{\downarrow \downarrow}=\operatorname{Tr}\left[\boldsymbol{\Gamma}_{L}^{\downarrow \downarrow} \mathbf{G}^{\downarrow \downarrow} \boldsymbol{\Gamma}_{R}^{\downarrow \downarrow}\left(\mathbf{G}^{\downarrow \downarrow}\right)^{\dagger}\right] .
\end{aligned}
$$

All quantities needed for the transmission function are calculated as in ARTAIOs. 11 The Green's function $\mathbf{G}$ is calculated as

$$
\mathbf{G}=\frac{1}{\left(E \mathbf{S}-\mathbf{H}_{C}-\mathbf{\Sigma}_{L}-\mathbf{\Sigma}_{R}\right)}
$$

and the coupling matrices $\boldsymbol{\Gamma}_{X}$ as

$$
\Gamma_{\boldsymbol{X}}=-2 \operatorname{Im}\left(\boldsymbol{\Sigma}_{\boldsymbol{X}}\right)
$$


The self energies $\boldsymbol{\Sigma}_{\boldsymbol{X}}$ can be calculated using the overlap and Hamiltonian matrix elements between the central region and the electrode $X$ as

$$
\boldsymbol{\Sigma}_{X}=\left(E \mathbf{S}_{X C}-\mathbf{H}_{X C}\right)^{\dagger} \mathbf{g}_{X}\left(E \mathbf{S}_{X C}-\mathbf{H}_{X C}\right)
$$

where $\mathbf{g}_{\mathbf{X}}$ is the Green's function of the electrode $X$. The Green's function is approximated within the wide-band limit, assuming a constant local density of states LDOS ${ }^{\text {const }}$

$$
\left(\mathbf{g}_{X}\right)_{i j}=-\mathrm{i} \pi \operatorname{LDOS}^{\text {const }} \delta_{i j}
$$

The value for the constant LDOS was set to $0.036 \mathrm{eV}^{-1}$ as calculated by DFT for the $6 s$ band of gold. $\frac{86}{6}$ This leads to purely imaginary self-energies. Nevertheless, our results are transferable to general self-energies, since if necessary their real part could be incorporated in the central-region Hamiltonian as a shift of effective single-particle energies caused by the interactions with the electrodes.

The matrices $\mathbf{S}_{X C}$ and $\mathbf{H}_{X C}$ originate from a scalar-relativistic calculation without spinorbit coupling which enforces a collinear spin orientation on the electrodes to achieve a clear definition of spin polarization in the transmission (see above). Therefore, while $\mathbf{H}_{C}$ has the form

$$
\mathbf{H}_{C}=\left(\begin{array}{cc}
\mathbf{H}_{C}^{\uparrow \uparrow} & \mathbf{H}_{C}^{\uparrow \downarrow} \\
\mathbf{H}_{C}^{\downarrow \uparrow} & \mathbf{H}_{C}^{\downarrow \downarrow}
\end{array}\right) .
$$

due to the spin-orbit coupling the "spin-flip" blocks of the self-energies $(\mathrm{X}=\mathrm{L}, \mathrm{R})$ are zero,

$$
\Sigma_{X}=\left(\begin{array}{cc}
\Sigma_{X}^{\uparrow \uparrow} & 0 \\
0 & \Sigma_{X}^{\downarrow \downarrow}
\end{array}\right)
$$


The used matrices therefore are extracted from the results of different DFT calculations: $\mathbf{H}_{C}$ from a calculation including spin-orbit coupling, $\mathbf{S}_{X C}$ and $\mathbf{H}_{X C}$ from a scalar-relativistic calculation.

\section{Derivation of the absence of polarization for closed- shell molecules in the Landauer approach without considering spin-orbit coupling}

Without spin-orbit coupling, $\mathbf{H}_{C}$ for a closed-shell system is symmetric, ${ }^{87}$ the spin-up and spin-down blocks are the same, and the off-diagonal blocks are zero,

$$
\begin{aligned}
\mathbf{H}_{C} & =\mathbf{H}_{C}^{T} \\
\mathbf{H}_{C}^{\uparrow \uparrow} & =\mathbf{H}_{C}^{\downarrow \downarrow} \\
\mathbf{H}_{C}^{\uparrow \downarrow} & =\mathbf{H}_{C}^{\downarrow \uparrow}=0
\end{aligned}
$$

Since the overlap matrix ${ }^{87}$ and self-energies are also symmetric, the Green's function must be symmetric as well,

$$
\mathbf{G}=\left(E \mathbf{S}-\mathbf{H}_{C}-\boldsymbol{\Sigma}_{\boldsymbol{L}}-\boldsymbol{\Sigma}_{\boldsymbol{R}}\right)^{-1}=\left(E \mathbf{S}^{T}-\mathbf{H}_{C}^{T}-\boldsymbol{\Sigma}_{\boldsymbol{L}}^{T}-\boldsymbol{\Sigma}_{\boldsymbol{R}}^{T}\right)^{-1}=\mathbf{G}^{T}
$$

By using the symmetry of the Green's function and the fact that for a closed-shell systems $\Gamma^{\uparrow \uparrow}=\Gamma^{\downarrow \downarrow}$ and $\mathbf{G}^{\uparrow \uparrow}=\mathbf{G}^{\downarrow \downarrow}$, it can be shown that

$$
T_{R L}^{\uparrow \uparrow}=T_{L R}^{\uparrow \uparrow}=T_{L R}^{\downarrow \downarrow}=T_{R L}^{\downarrow \downarrow}
$$

Therefore, no spin-dependent transmission and thus no spin polarization can occur without spin-orbit coupling in our approach. This relations can be proven by reformulating the 
transmission function as the transpose of the matrix which is traced to calculate the spindependent transmission functions. The trace of a matrix and the trace of its transpose are the same, therefore we can write

$$
\begin{aligned}
& T_{R L}^{\uparrow \uparrow}=\operatorname{Tr}\left[\left(\boldsymbol{\Gamma}_{L}^{\uparrow \uparrow} \mathbf{G}^{\uparrow \uparrow} \boldsymbol{\Gamma}_{R}^{\uparrow \uparrow}\left(\mathbf{G}^{\uparrow \uparrow}\right)^{\dagger}\right)^{T}\right] \\
& T_{R L}^{\downarrow \downarrow}=\operatorname{Tr}\left[\left(\boldsymbol{\Gamma}_{L}^{\downarrow \downarrow} \mathbf{G}^{\downarrow \downarrow} \boldsymbol{\Gamma}_{R}^{\downarrow \downarrow}\left(\mathbf{G}^{\downarrow \downarrow}\right)^{\dagger}\right)^{T}\right] .
\end{aligned}
$$

This can be rewritten as

$$
\begin{aligned}
& T_{R L}^{\uparrow \uparrow}=\operatorname{Tr}\left[\left(\left(\boldsymbol{\Gamma}_{L}^{\uparrow \uparrow}\right)^{T}\left(\left(\mathbf{G}^{\uparrow \uparrow}\right)^{\dagger}\right)^{T}\left(\boldsymbol{\Gamma}_{R}^{\uparrow \uparrow}\right)^{T}\left(\mathbf{G}^{\uparrow \uparrow}\right)^{T}\right]\right. \\
& T_{R L}^{\downarrow \downarrow}=\operatorname{Tr}\left[\left(\boldsymbol{\Gamma}_{L}^{\downarrow \downarrow}\right)^{T}\left(\left(\mathbf{G}^{\downarrow \downarrow}\right)^{\dagger}\right)^{T}\left(\boldsymbol{\Gamma}_{R}^{\downarrow \downarrow}\right)^{T}\left(\mathbf{G}^{\downarrow \downarrow}\right)^{T}\right]
\end{aligned}
$$

(where it has been exploited that $(\mathbf{A B})^{T}=\mathbf{B}^{T} \mathbf{A}^{T}$ and that the order of matrices can be exchanged cyclically within a trace). We can now use the relation $\mathbf{G}=\mathbf{G}^{T}$ as well as the fact that $\Gamma_{X}$ is symmetric to get

$$
\begin{aligned}
& T_{R L}^{\uparrow \uparrow}=\operatorname{Tr}\left[\boldsymbol{\Gamma}_{L}^{\uparrow \uparrow}\left(\mathbf{G}^{\uparrow \uparrow}\right)^{\dagger} \boldsymbol{\Gamma}_{R}^{\uparrow \uparrow} \mathbf{G}^{\uparrow \uparrow}\right]=T_{L R}^{\uparrow \uparrow} \\
& T_{R L}^{\downarrow \downarrow}=\operatorname{Tr}\left[\boldsymbol{\Gamma}_{L}^{\downarrow \downarrow}\left(\mathbf{G}^{\downarrow \downarrow}\right)^{\dagger} \boldsymbol{\Gamma}_{R}^{\downarrow \downarrow} \mathbf{G}^{\downarrow \downarrow}\right]=T_{L R}^{\downarrow \downarrow} .
\end{aligned}
$$

Since we are interested in closed-shell systems we can also assume that $\mathbf{G}^{\uparrow \uparrow}=\mathbf{G}^{\downarrow \downarrow}$ and $\Gamma_{X}^{\uparrow \uparrow}=\Gamma_{X}^{\downarrow \downarrow}$ we arrive at Equation 75 . 


\section{E Derivation of the possibility of polarization for closed- shell molecules in the Landauer approach by consid- ering spin-orbit coupling}

To derive spin-orbit coupling as responsible for polarization within the Landauer approach for closed-shell molecules, we follow the same procedure as in the previous section. This derivation shows how spin polarization is induced by spin-orbit coupling, but does not discuss the magnitude of the effect.

Due to time-reversal symmetry, the blocks of $\mathbf{H}_{C}$ are related as ${ }^{76}$

$$
\begin{aligned}
\mathbf{H}_{C} & =\mathbf{H}_{C}^{\dagger} \\
\mathbf{H}_{C}^{\uparrow \uparrow} & =\left(\mathbf{H}_{\mathbf{C}^{\downarrow \downarrow}}\right)^{*} \\
\mathbf{H}_{C}^{\uparrow \downarrow} & =-\left(\mathbf{H}_{C}^{\downarrow \uparrow}\right)^{*} .
\end{aligned}
$$

Using Equation (82), Equations (83) and (84) can be reformulated as

$$
\begin{aligned}
& \mathbf{H}_{C}^{\uparrow \uparrow}=\left(\mathbf{H}_{C}^{\downarrow \downarrow}\right)^{*}=\left(\left[\mathbf{H}_{C}^{\downarrow \downarrow}\right]^{*}\right)^{\dagger}=\left(\mathbf{H}_{C}^{\downarrow \downarrow}\right)^{\mathrm{T}} \\
& \mathbf{H}_{C}^{\uparrow \downarrow}=-\left(\mathbf{H}_{C}^{\downarrow \uparrow}\right)^{*}=-\left(\left[\mathbf{H}_{C}^{\uparrow \downarrow}\right]^{*}\right)^{\dagger}=-\left(\mathbf{H}_{C}^{\uparrow \downarrow}\right)^{\mathrm{T}} .
\end{aligned}
$$

Upon combining $\mathbf{H}_{C}$ with $\boldsymbol{\Sigma}_{L}$ and $\boldsymbol{\Sigma}_{R}$ the relation $\mathbf{H}_{C}^{\uparrow \uparrow}=\left(\mathbf{H}_{C}^{\Downarrow \downarrow}\right)^{\mathrm{T}}$ remains,

$$
\begin{aligned}
\mathbf{H}_{C}^{\uparrow \uparrow}-\boldsymbol{\Sigma}^{\uparrow \uparrow} & =\operatorname{Re}\left(\mathbf{H}_{C}^{\uparrow \uparrow}\right)+\operatorname{Im}\left(\mathbf{H}_{C}^{\uparrow \uparrow}\right)-\operatorname{Im}\left(\boldsymbol{\Sigma}^{\uparrow \uparrow}\right) \\
& =\operatorname{Re}\left(\mathbf{H}_{C}^{\downarrow \downarrow}\right)^{T}+\operatorname{Im}\left(\mathbf{H}_{C}^{\downarrow \downarrow}\right)^{T}-\operatorname{Im}\left(\boldsymbol{\Sigma}^{\downarrow \downarrow}\right)^{T}=\left(\mathbf{H}_{C}^{\downarrow \downarrow}-\boldsymbol{\Sigma}^{\downarrow \downarrow}\right)^{T},
\end{aligned}
$$


while the relation $\mathbf{H}_{C}^{\uparrow \uparrow}=\left(\mathbf{H}_{C}^{\downarrow \downarrow}\right)^{*}$ does not,

$$
\begin{aligned}
\mathbf{H}_{C}^{\uparrow \uparrow}-\boldsymbol{\Sigma}^{\uparrow \uparrow} & =\operatorname{Re}\left(\mathbf{H}_{C}^{\uparrow \uparrow}\right)+\operatorname{Im}\left(\mathbf{H}_{C}^{\uparrow \uparrow}\right)-\operatorname{Im}\left(\boldsymbol{\Sigma}^{\uparrow \uparrow}\right) \\
& \neq \operatorname{Re}\left(\mathbf{H}_{C}^{\downarrow \downarrow}\right)-\operatorname{Im}\left(\mathbf{H}_{C}^{\downarrow \downarrow}\right)+\operatorname{Im}\left(\boldsymbol{\Sigma}^{\downarrow \downarrow}\right)=\left(\mathbf{H}_{C}^{\downarrow \downarrow}-\boldsymbol{\Sigma}^{\downarrow \downarrow}\right)^{*} .
\end{aligned}
$$

Therefore, the blocks of $\mathbf{G}$ are only related as

$$
\begin{aligned}
& \mathbf{G}^{\uparrow \uparrow}=\left(\mathbf{G}^{\downarrow \downarrow}\right)^{\mathrm{T}}, \\
& \mathbf{G}^{\uparrow \downarrow}=-\left(\mathbf{G}^{\uparrow \downarrow}\right)^{\mathrm{T}} .
\end{aligned}
$$

We follow the same procedure as for the transmission function without spin-orbit coupling, reformulating the transmission function as

$$
\begin{aligned}
& T_{R L}^{\uparrow \uparrow}=\operatorname{Tr}\left[\left(\boldsymbol{\Gamma}_{L}^{\uparrow \uparrow}\right)^{T}\left(\left(\mathbf{G}^{\uparrow \uparrow}\right)^{\dagger}\right)^{T}\left(\boldsymbol{\Gamma}_{R}^{\uparrow \uparrow}\right)^{T}\left(\mathbf{G}^{\uparrow \uparrow}\right)^{T}\right] \\
& T_{R L}^{\downarrow \uparrow}=\operatorname{Tr}\left[\left(\boldsymbol{\Gamma}_{L}^{\uparrow \uparrow}\right)^{T}\left(\left(\mathbf{G}^{\downarrow \uparrow}\right)^{\dagger}\right)^{T}\left(\boldsymbol{\Gamma}_{R}^{\downarrow \downarrow}\right)^{T}\left(\mathbf{G}^{\downarrow \uparrow}\right)^{T}\right] \\
& T_{R L}^{\uparrow \downarrow}=\operatorname{Tr}\left[\left(\boldsymbol{\Gamma}_{L}^{\downarrow \downarrow}\right)^{T}\left(\left(\mathbf{G}^{\uparrow \downarrow}\right)^{\dagger}\right)^{T}\left(\boldsymbol{\Gamma}_{R}^{\uparrow \uparrow}\right)^{T}\left(\mathbf{G}^{\uparrow \downarrow}\right)^{T}\right] \\
& T_{R L}^{\downarrow \downarrow}=\operatorname{Tr}\left[\left(\boldsymbol{\Gamma}_{L}^{\downarrow \downarrow}\right)^{T}\left(\left(\mathbf{G}^{\downarrow \downarrow}\right)^{\dagger}\right)^{T}\left(\boldsymbol{\Gamma}_{R}^{\downarrow \downarrow}\right)^{T}\left(\mathbf{G}^{\downarrow \downarrow}\right)^{T}\right] .
\end{aligned}
$$

Making use of the symmetries in Equation 93 and 95 as well as of the relation $\boldsymbol{\Gamma}_{X}^{\uparrow \uparrow}=\Gamma_{X}^{\downarrow \downarrow}$, we get

$$
\begin{aligned}
& T_{R L}^{\uparrow \uparrow}=\operatorname{Tr}\left[\boldsymbol{\Gamma}_{L}^{\downarrow \downarrow}\left(\mathbf{G}^{\downarrow \downarrow}\right)^{\dagger} \boldsymbol{\Gamma}_{R}^{\downarrow \downarrow} \mathbf{G}^{\downarrow \downarrow}\right]=T_{L R}^{\downarrow \downarrow} \\
& T_{R L}^{\downarrow \uparrow}=\operatorname{Tr}\left[\boldsymbol{\Gamma}_{L}^{\downarrow \downarrow}\left(\mathbf{G}^{\downarrow \uparrow}\right)^{\dagger} \boldsymbol{\Gamma}_{R}^{\uparrow \uparrow} \mathbf{G}^{\downarrow \uparrow}\right]=T_{L R}^{\downarrow \uparrow} \\
& T_{R L}^{\uparrow \downarrow}=\operatorname{Tr}\left[\boldsymbol{\Gamma}_{L}^{\uparrow \uparrow}\left(\mathbf{G}^{\uparrow \downarrow}\right)^{\dagger} \boldsymbol{\Gamma}_{R}^{\downarrow \downarrow} \mathbf{G}^{\uparrow \downarrow}\right]=T_{L R}^{\uparrow \downarrow} \\
& T_{R L}^{\downarrow \downarrow}=\operatorname{Tr}\left[\boldsymbol{\Gamma}_{L}^{\uparrow \uparrow}\left(\mathbf{G}^{\uparrow \uparrow}\right)^{\dagger} \boldsymbol{\Gamma}_{R}^{\uparrow \uparrow} \mathbf{G}^{\uparrow \uparrow}\right]=T_{L R}^{\uparrow \uparrow} .
\end{aligned}
$$

Since as shown in Equation (16), the symmetry of $\mathbf{G}$ is broken due to the self energies, we 
generally have to assume that

$$
T_{R L}^{\sigma \sigma^{\prime}} \neq T_{L R}^{\sigma \sigma^{\prime}} .
$$

Therefore, we can only state the relations stated in Eqs. (96) through (99). which opens up the possibility for spin polarization withing the Landauer transport regime.

\section{F Manual for reading the matrices}

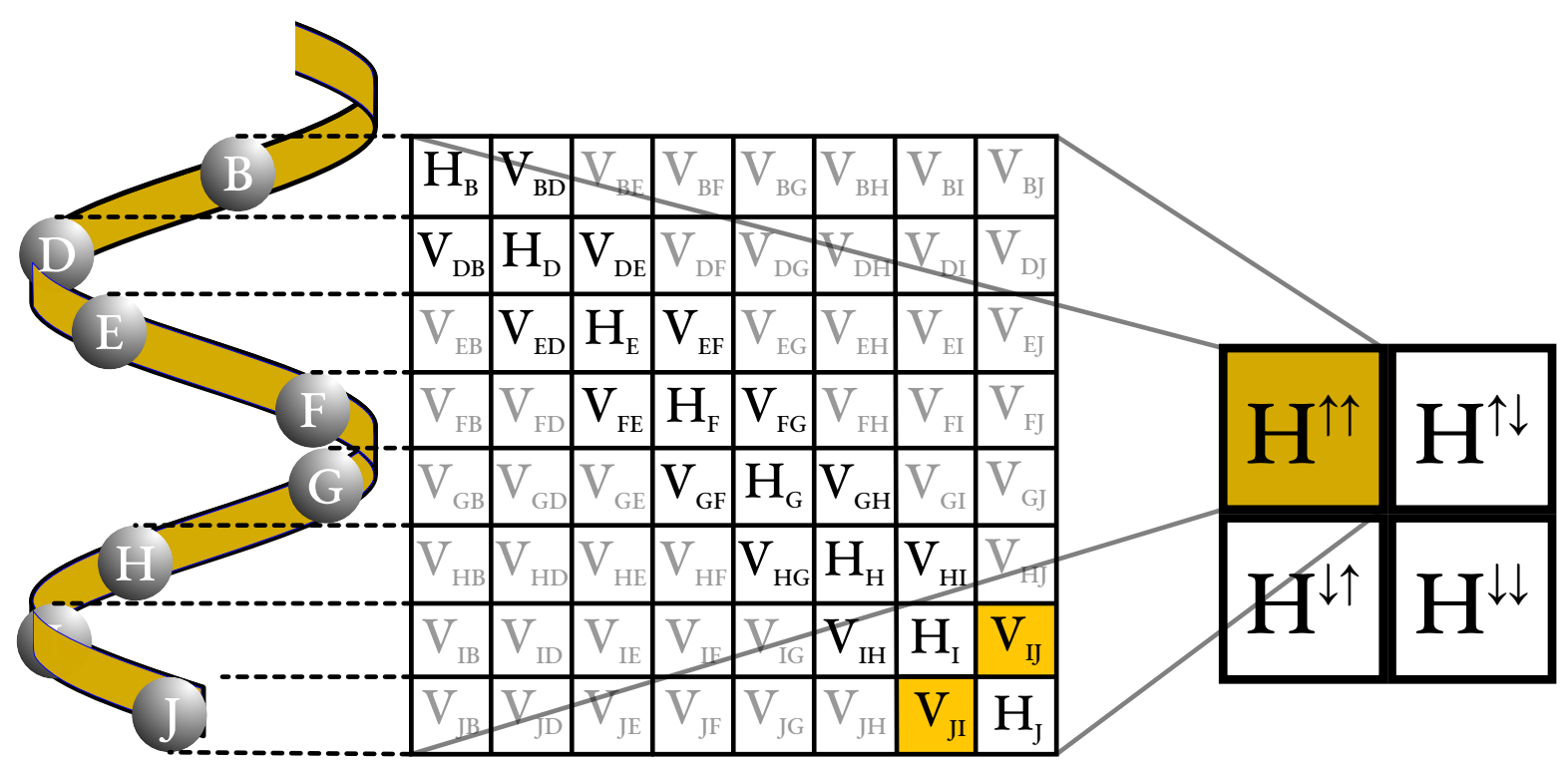

Figure 8: Structure of the matrices of the cumulene molecules, analyzed in this work. The effective single-particle Hamiltonian matrix consists of four spin-dependent blocks $\boldsymbol{H}^{\sigma \sigma^{\prime}}$ and we are focused on the $\boldsymbol{H}^{\uparrow \uparrow}$ block during this work. $\boldsymbol{H}^{\uparrow \uparrow}$ consists of diagonal-blocks $\left(\boldsymbol{H}_{I}\right)$ describing the spin-conserving coupling of the atomic orbitals located on atom $I$, and of offdiagonal blocks $\left(\boldsymbol{V}_{I, J}\right)$ describing the coupling of all atomic orbitals located on atom $I$ with all atomic orbitals located on atom $J$. The diagonal blocks are sorted along the helix-path. To get a better understanding of the origin of the spin-polarization of transmitted electrons we focus on a single pair of nearest-neighbour couplings $\left(\boldsymbol{V}_{I,(I+1)} / \boldsymbol{V}_{(I+1), I}\right)$. Those elements can be interpreted as transitions amplitudes along and against transport direction.

The matrices are structured as follows: The matrices consist of a diagonal and off-diagonal blocks. Each diagonal block $\boldsymbol{H}_{I}$ can be attributed to an atom $I$ containing the couplings of between the atomic orbitals located on atom $I$. The off-diagonal blocks $\boldsymbol{V}_{I, J}$ can be 
attributed to the coupling of atom $I$ with atom $J$, containing the coupling of all orbitals located on atom $I$ with all orbitals located in atom $J$. The blocks are sorted in ascending direction.

Each spin-dependent block $\boldsymbol{H}^{\sigma \sigma^{\prime}}$ consists of such a block matrix. We are focused on a a single pair of nearest-neighbour coupling blocks $\boldsymbol{V}_{I,(I+1)} / \boldsymbol{V}_{(I+1), I}$ of $\boldsymbol{H}^{\sigma \sigma^{\prime}}$ (see Figure 8).

\section{References}

(1) Naaman, R.; Paltiel, Y.; Waldeck, D. H. Chiral molecules and the electron spin. Nat. Rev. Chem. 2019, 3, 250-260.

(2) Abendroth, J. M.; Cheung, K. M.; Stemer, D. M.; Hadri, M. S. E.; Zhao, C.; Fullerton, E. E.; Weiss, P. S. Spin-Dependent Ionization of Chiral Molecular Films. J. Am. Chem. Soc. 2019, 141, 3863-3874.

(3) Mtangi, W.; Tassinari, F.; Vankayala, K.; Jentzsch, A. V.; Adelizzi, B.; Palmans, A. R. A.; Fontanesi, C.; Meijer, E. W.; Naaman, R. Control of Electrons Spin Eliminates Hydrogen Peroxide Formation During Water Splitting. J. Am. Chem. Soc. 2017, 139, $2794-2798$.

(4) Mtangi, W.; Kiran, V.; Fontanesi, C.; Naaman, R. Role of the Electron Spin Polarization in Water Splitting. J. Phys. Chem. Lett. 2015, 6, 4916-4922.

(5) Gazzotti, M.; Arnaboldi, S.; Grecchi, S.; Giovanardi, R.; Cannio, M.; Pasquali, L.; Giacomino, A.; Abollino, O.; Fontanesi, C. Spin-dependent electrochemistry: Enantioselectivity driven by chiral-induced spin selectivity effect. Electrochim. Acta 2018, 286, $271-278$.

(6) Mondal, P. C.; Mtangi, W.; Fontanesi, C. ChiroSpintronics: SpinDependent Electrochemistry and Water Splitting Using Chiral Molecular Films. small methods 2018, 2, 1700313. 
(7) Santos, J. I.; Rivilla, I.; o P. Cossio, F.; Matxain, J. M.; Grzelczak, M.; Mazinani, S. K. S.; Ugalde, J. M.; Mujica, V. Chirality-Induced Electron Spin Polarization and Enantiospecific Response in Solid-State Cross-Polarization Nuclear Magnetic Resonance. ACS Nano 2018, 12, 11426-11433.

(8) Xie, Z.; Markus, T. Z.; Cohen, S. R.; Vager, Z.; Gutierrez, R.; Naaman, R. Spin Specific Electron Conduction through DNA Oligomers. Nano Lett. 2011, 11, 4652-4655.

(9) Zwang, T. J.; Hürlimann, S.; Hill, M. G.; Barton, J. K. Helix-Dependent Spin Filtering through the DNA Duplex. J. Am. Chem. Soc. 2016, 138, 15551-15554.

(10) Göhler, B.; Hamelbeck, V.; Markus, T. Z.; Kettner, M.; Hanne, G. F.; Vager, Z.; Naaman, R.; Zacharias, H. Spin selectivity in electron transmission through self-assembled monolayers of double-stranded DNA. Science 2011, 331, 894-897.

(11) Mishra, D.; Markus, T. Z.; Naaman, R.; Kettner, M.; Göhler, B.; Zacharias, H.; Friedman, N.; Sheves, M.; Fontanesi, C. Spin-dependent electron transmission through bacteriorhodopsin embedded in purple membrane. Proc. Natl. Acad. Sci. 2013, 110, 1487214876.

(12) Aragones, A. C.; Medina, E.; Ferrer-Huerta, M.; Gimeno, N.; Teixido, M.; Palma, J. L.; Tao, N.; Ugalde, J. M.; Giralt, E.; Diez-Perez, I.; Mujica, V. Measuring the SpinPolarization Power of a Single Chiral Molecule. small 2017, 13, 1602519.

(13) Kettner, M.; Göhler, B.; Zacharias, H.; Mishra, D.; Kiran, V.; Naaman, R.; Fontanesi, C.; Waldeck, D. H.; Sek, S.; Pawlowski, J.; Juhaniewicz, J. Spin Filtering in Electron Transport Through Chiral Oligopeptides. J. Phys. Chem. C 2015, 119, 14542-14547.

(14) Kettner, M.; Maslyuk, V. V.; Nürenberg, D.; Seibel, J.; Gutierrez, R.; Cuniberti, G.; Ernst, K.-H.; Zacharias, H. Chirality-Dependent Electron Spin Filtering by Molecular Monolayers of Helicenes. J. Phys. Chem. Lett. 2018, 9, 2025-2030. 
(15) Kiran, V.; Mathew, S. P.; Cohen, S. R.; Delgado, I. H.; Lacour, J.; Naaman, R. Helicenes - A New Class of Organic Spin Filter. Adv. Mater. 2016, 28, 1957-1962.

(16) Guo, A.-M.; Pan, T.-R.; Fang, T.-F.; Xie, X. C.; Sun, Q.-F. Spin selectivity effect in achiral molecular systems. Phys. Rev. B 2016, 94, 165409.

(17) Brandt, J. R.; Salerno, F.; Fuchter, M. J. The added value of small-molecule chirality in technological applications. Nat. Rev. Chem. 2017, 1, 0045.

(18) Yeganeh, S.; Ratner, M. A.; Medina, E.; Mujica, V. Chiral electron transport: Scattering through helical potentials. J. Chem. Phys. 2009, 131, 014707.

(19) Varela, S.; Mujica, V.; Medina, E. Effective spinorbit couplings in an analytical tightbinding model of DNA: spin filtering and chiral spin transport. Phys. Rev. B 2016, 93, 155436.

(20) Medina, E.; Lopez, F.; Ratner, M. A.; Mujica, V. Chiral molecular films as electron polarizers and polarization modulators. Eur. Phys. Lett. 2012, 99, 17006.

(21) Gutierrez, R.; Díaz, E.; Naaman, R.; Cuniberti, G. Spin-selective transport through helical molecular systems. Phys. Rev. B 2012, 85, 081404-081407.

(22) Gutierrez, R.; Díaz, E.; Gaul, C.; Brumme, T.; Domínguez-Adame, F.; Cuniberti, G. Modeling Spin Transport in Helical Fields: Derivation of an Effective Low-Dimensional Hamiltonian. J. Phys. Chem. C 2013, 117, 22276-22284.

(23) Maslyuk, V. V.; Gutierrez, R.; Dianat, A.; Mujica, V.; Cuniberti, G. Enhanced Magnetoresistance in Chiral Molecular Junctions. J. Chem. Phys. Lett. 2018, 9, 5453-5459.

(24) Guo, A.-M.; Sun, Q.-f. Spin-selective transport of electrons in DNA double helix. Phys. Rev. Lett. 2012, 108, 218102.

(25) Guo, A.-M.; Sun, Q.-F. Spin-dependent electron transport in protein-like single-helical molecules. Proc. Natl. Acad. Sci. 2014, 111, 11658-11662. 
(26) Dalum, S.; Hedegård, P. Theory of Chiral Induced Spin Selectivity. Nano Lett. 2019, $19,5253-5259$.

(27) Winkler, R., Ed. Spin-Orbit Coupling Effects in Two-Dimensional Electron and Hole Systems; Springer: Berlin, 2003.

(28) Forster, F.; Bendounan, A.; Reinert, F.; Grigoryan, V. G.; Springborg, M. The Shockley-type surface state on $\mathrm{Ar}$ covered $\mathrm{Au}(111)$ : High resolution photoemission results and the description by slab-layer DFT calculations. Surf. Sci. 2007, 601, 55955604 .

(29) LaShell, S.; McDougall, B. A.; Jensen, E. Spin Splitting of an Au(111) Surface State Band Observed with Angle Resolved Photoelectron Spectroscopy. Phys. Rev. Lett. 1996, 777, 3419-3422.

(30) Moayed, M. M. R.; Bielewicz, T.; Zöllner, M. S.; Herrmann, C.; Klinke, C. On the way to colloidal spintronics Rashba spinorbit interaction in PbS nanosheets. Nat. Commun. $2017,8,15721$.

(31) Varela, S.; Mujica, V.; Medina, E. Spin-orbit Coupling Modulation in DNA by Mechanical Deformations. Chimia 2018, 72, 411-417.

(32) Zotti, L. A.; Carlos, J. Electron Transport Through Homopeptides: Are They Really Good Conductors? ACS Omega 2018, 3, 3778-3785.

(33) Sepunaru, L.; Refaely-Abramson, S.; Lovrincic, R.; Gavrilov, Y.; Agrawal, P.; Levy, Y.; Kronik, L.; Pecht, I.; Sheves, M.; Cahen, D. Electronic Transport via Homopeptides: The Role of Side Chains and Secondary Structure. J. Am. Chem. Soc. 2015, 137, 9617-9626.

(34) Xiang, L.; Palma, J. L.; Bruot, C.; Mujica, V.; Ratner, M. A.; Tao, N. Intermediate tunnelling-hopping regime in DNA charge transport. Nat. Chem. 2015, 7, 221-226. 
(35) Mujica, V.; Kemp, M.; Ratner, M. A. Electron conduction in molecular wires. I. A scattering formalism. J. Chem. Phys. 1994, 101, 6849-6855.

(36) Petersen, L.; Hedegård, P. A simple tight-binding model of spin-orbit splitting of spderived surface states. Surf. Sci. 2000, 459, 49-56.

(37) Caroli, C.; Combescot, R.; Nozieres, P.; Saint-James, D. Direct calculation of the tunneling current. J. Phys. C: Solid State Phys. 1971, 4, 916-929.

(38) Wilhelm, J.; Walz, M.; Evers, F. Ab initio spin-flip conductance of hydrogenated graphene nanoribbons: Spin-orbit interaction and scattering with local impurity spins. Phys. Rev. B 2015, 92, 014405.

(39) Bhattacharya, S.; Akande, A.; Sanvito, S. Spin transport properties of triarylaminebased nanowires. Chem. Commun. 2014, 50, 6626-6629.

(40) Herrmann, C.; Solomon, G. C.; Subotnik, J. E.; Mujica, V.; Ratner, M. A. Ghost transmission: How large basis sets can make electron transport calculations worse. J. Chem. Phys. 2010, 132, 024103.

(41) Deffner, M.; Gross, L.; Steenbock, T.; Voigt, B. A.; Solomon, G. C.; Herrmann, C. ARTAIOS - a transport code for postprocessing quantum chemical electronic structure calculations, available via https://www.chemie.unihamburg.de/institute/ac/arbeitsgruppen/herrmann/software/artaios.html. 2009-2019.

(42) Bardarson, J. H. A proof of the Kramers degeneracy of transmission eigenvalues from antisymmetry of the scattering matrix. J. Phys. A: Math. Theor. 2008, 41, 405203.

(43) Rashba, E. I. Spin currents in thermodynamic equilibrium: The challenge of discerning transport currents. Phys. Rev. B 2003, 68, 241315(R).

(44) Kiselev, A. A.; Kim, K. W. Prohibition of equilibrium spin currents in multiterminal ballistic devices. Phys. Rev. B 2005, 71, 153315. 
(45) Yang, X.; Bosma, T.; van Wees, B. J.; van der Wal, C. Circuit-model analysis for spintronic devices with chiral molecules as spin injectors. Phys. Rev. B 2019, 99, 214428.

(46) Armbruster, M. K.; Weigend, F.; Wüllen, C.; Klopper, W. Self-consistent treatment of spinorbit interactions with efficient HartreeFock and density functional methods. Phys. Chem. Chem. Phys. 2008, 10, 1748-1756.

(47) van Wüllen, C. A Quasirelativistic Two-component Density Functional and HartreeFock Program. Z. Phys. Chem. 2010, 224, 413.

(48) Di Ventra, M. Electrical Transport in Nanoscale Systems; Cambridge University Press: New York, 2008.

(49) Nürenberg, D.; Zacharias, H. Evaluation of spin-flip scattering in chirality-induced spin selectivity using the Riccati equation. Phys. Chem. Chem. Phys. 2019, 21, 3761-3770.

(50) Lopez, A.; Colmenarez, L.; Peralta, M.; Mireles, F.; Medina, E. Proximity-induced spin-orbit effects in graphene on Au. Phys. Rev. B 2019, 99, 085411.

(51) Marchenko, D.; Varykhalov, A.; Scholz, M. R.; Bihlmayer, G.; Rashba, E. I.; Rybkin, A.; Shikin, A. M.; Rader, O. Nat. Commun. 2012, 3, 1232.

(52) Gersten, J.; Kaasbjerg, K.; Nitzan, A. Induced spin filtering in electron transmission through chiral molecular layers adsorbed on metals with strong spin-orbit coupling. J. Chem. Phys. 2013, 139, 114111.

(53) Varela, S.; Zambrano, I.; Mujica, V.; Berche, B.; Medina, E. Spin-orbit interaction and spin selectivity for tunneling electron transfer in DNA. 2019, to be submitted.

(54) Taylor, J. R. Scattering Theory: The Quantum Theory of Nonrelativistic Collision; Dover Publications Inc: New York, 2006.

(55) Banerjee-Ghosh, K.; Ben Dor, O.; Tassinari, F.; Capua, E.; Yochelis, S.; Capua, A.; Yang, S.-H.; Parkin, S. S. P.; Sarkar, S.; Kronik, L.; Baczewski, L. T.; Naaman, R.; 
Paltiel, Y. Separation of enantiomers by their enantiospecific interaction with achiral magnetic substrates. Science 2018, 360, 1331-1334.

(56) Geyer, M.; Gutierrez, R.; Mujica, V.; Cuniberti, G. Chirality-Induced Spin Selectivity in a Coarse-Grained Tight-Binding Model for Helicene. J. Phys. Chem. C 2019, just accepted, DOI 10.1021/acs.jpcc.9b07764.

(57) Büttiker, M. Role of quantum coherence in series resistors. Phys. Rev. B 1986, 333, 3020-3026.

(58) Cattena, C. J.; Fernandez-Alcazar, L. J.; Bustos-Marun, R. A.; Nozaki, D.; Pastawski, H. M. Generalized multi-terminal decoherent transport: recursive algorithms and applications to SASER and giant magnetoresistance. J. Phys.: Condens. Matter 2014, 26, 345304.

(59) Nozaki, D.; Bustos-Marun, R.; Cattena, C. J.; Cuniberti, G.; Pastawski, H. M. An efficient coarse-grained approach for the electron transport through large molecular systems under dephasing environment. Eur. Phys. J. B 2016, 89, 102.

(60) Nozaki, D.; da Rocha, C. G.; Pastawski, H. M.; Cuniberti, G. Disorder and dephasing effects on electron transport through conjugated molecular wires in molecular junctions. Phys. Rev. B 2012, 85, 155327.

(61) Bandyopadhyay, M.; Segal, D. Quantum heat transfer in harmonic chains with selfconsistent reservoirs: Exact numerical simulations. Phys. Rev. E 2011, 84, 011151.

(62) Matityahu, A.; Utsumi, Y.; Aharony, A.; Entin-Wohlman, O.; Balseiro, C. A. Spindependent transport through a chiral molecule in the presence of spin-orbit interaction and nonunitary effects. Phys. Rev. B 2016, 93, 075407.

(63) TURBOMOLE V7.1, a development of University of Karlsruhe and Forschungszen- 
trum Karlsruhe GmbH, 1989-2007, TURBOMOLE GmbH, since 2007; available from http://www.turbomole.com. 2016.

(64) Ahlrichs, R.; Bär, M.; Häser, M.; Horn, H.; Kölmel, C. Electronic structure calculations on workstation computers: The program system turbomole. Chem. Phys. Lett. 1989, 162, $165-169$.

(65) Treutler, O.; Ahlrichs, R. Efficient molecular numerical integration schemes. J. Chem. Phys. 1995, 102, 346-354.

(66) Von Arnim, M.; Ahlrichs, R. Performance of parallel TURBOMOLE for density functional calculations. J. Comput. Chem. 1998, 19, 1746-1757.

(67) Perdew, J. P.; Burke, K.; Wang, Y. Generalized gradient approximation for the exchange-correlation hole of a many-electron system. Phys. Rev. B 1996, 54, 1653316539.

(68) Perdew, J. P.; Burke, K.; Ernzerhof, M. Generalized Gradient Approximation Made Simple. Phys. Rev. Lett. 1996, 77, 3865-3868.

(69) Schäfer, A.; Huber, C.; Ahlrichs, R. Fully optimized contracted Gaussian basis sets of triple zeta valence quality for atoms Li to Kr. J. Chem. Phys. 1994, 100, 5829-5835.

(70) Weigend, F.; Ahlrichs, R. Balanced basis sets of split valence, triple zeta valence and quadruple zeta valence quality for $\mathrm{H}$ to Rn: Design and assessment of accuracy. Phys. Chem. Chem. Phys. 2005, 7, 3297-3305.

(71) Grimme, S.; Antony, J.; Ehrlich, S.; Krieg, H. A consistent and accurate ab initio parametrization of density functional dispersion correction (DFT-D) for the 94 elements H-Pu. J. Chem. Phys. 2010, 132, 154104.

(72) Grimme, S.; Ehrlich, S.; Goerigk, L. Effect of the damping function in dispersion corrected density functional theory. J. Comput. Chem. 2011, 32, 1456-1465. 
(73) Eichkorn, K.; Treutler, O.; Öhm, H.; Häser, M.; Ahlrichs, R. Auxiliary basis sets to approximate Coulomb potentials. Chem. Phys. Lett. 1995, 240, $283-290$.

(74) Eichkorn, K.; Weigend, F.; Treutler, O.; Ahlrichs, R. Auxiliary basis sets for main row atoms and transition metals and their use to approximate Coulomb potentials. Theor. Chem. Acc. 1997, 97, 119-124.

(75) Weigend, F. Accurate Coulomb-fitting basis sets for H to Rn. Phys. Chem. Chem. Phys. 2006, 8, 1057-1065.

(76) Peng, D.; Middendorf, N.; Weigend, F.; Reiher, M. An efficient implementation of twocomponent relativistic exact-decoupling methods for large molecules. J. Chem. Phys. 2013, 138, 184105.

(77) Becke, A. D. Density-functional exchange-energy approximation with correct asymptotic behavior. Phys. Rev. A 1988, 38, 3098-3100.

(78) Lee, C.; Yang, W.; Parr, R. G. Development of the Colle-Salvetti correlation-energy formula into a functional of the electron density. Phys. Rev. B 1988, 37, 785-789.

(79) Becke, A. D. Densityfunctional thermochemistry. III. The role of exact exchange. $J$. Chem. Phys. 1993, 98, 5648-5652.

(80) Dyall, K. G. Interfacing relativistic and nonrelativistic methods. I. Normalized elimination of the small component in the modified Dirac equation. J. Chem. Phys. 1997, 106, 9618-9626.

(81) Kutzelnigg, W.; Liu, W. Quasirelativistic theory equivalent to fully relativistic theory. J. Chem. Phys. 2005, 123, 241102.

(82) Pollak, P.; Weigend, F. Segmented Contracted Error-Consistent Basis Sets of Doubleand Triple- Valence Quality for One- and Two-Component Relativistic All-Electron Calculations. J. Chem. Theory Comput. 2017, 13, 3696-3705. 
(83) Sherman, R. P.; Grinter, R. Transformation matrices for the rotation of real p, d, and f atomic orbitals. J. Mol. Struc.: THEOCHEM 1986, 135, 127 - 133.

(84) Mabbs, F. E.; Machin, D. J. Magnetism and Transition Metal Complexes; Dover Publications Inc: New York, 2008.

(85) Atkins, P.; Friedman, R. Molecular Quantum Mechanics, 5th ed.; Oxford University Press: New York, 2010; p 92.

(86) Papaconstantopoulos, D. A. Handbook of the Band Structure of Elemental Solids; Plenum: New York, 1986.

(87) Szabo, A.; Ostlund, N. Modern Quantum Chemistry: Introduction to Advanced Electronic Structure Theory; Dover Publications Inc: New York, 1996. 


\section{Graphical TOC Entry}
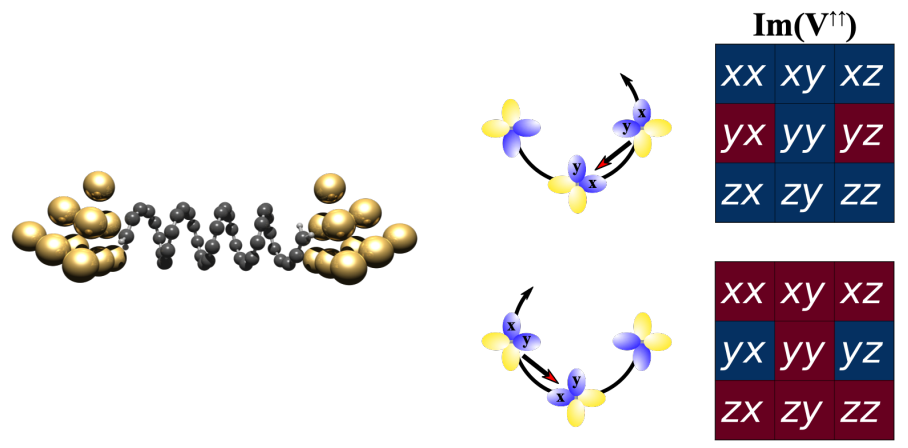